\title{
Traditional and novel methods to assess and prevent chemotherapy-related cardiac dysfunction noninvasively
}

\author{
Ronald G. Schwartz, MD, MS, FACC, FAHA, ABNM, FASNC, ${ }^{a}$ Diwakar Jain, MD, \\ FACC, FRCP, FASNC, ${ }^{b}$ and Eugene Storozynsky, MD, PhD, FACC ${ }^{a}$
}

The field of cardio-oncology is challenged to address an ever greater spectrum of cardiotoxicity associated with combination chemotherapy, greater dose intensity, extremes of age, and enhanced patient survival which exposes more protracted risk of developing congestive heart failure (CHF). Recent reports of chemotherapy-induced hypertension as a common adverse effect of angiogenesis inhibitors and immunosuppressants clarify the need for routine blood pressure (BP) monitoring and guideline-based management of hypertension as an integral strategy to preserve $\mathrm{LV}$ function. Serial monitoring of radionuclide left ventricular ejection fraction (LVEF) in adults and echocardiography in children continues to provide outcome based, cost-effective prevention of $\mathrm{CHF}$ in high risk patients receiving chemotherapy. To optimize treatment and monitoring strategies to eliminate late-onset $\mathrm{LV}$ dysfunction and CHF, traditional and novel candidate methods for assessment of chemotherapy-induced LV dysfunction are reviewed. These include serial assessment of $L V$ volume indices by gated SPECT ERNA and gated SPECT MPI, 3D echocardiography and contrast 2D echocardiography; longitudinal strain imaging, diastolic functional parameters, ${ }^{123}$ I-MIBG, ${ }^{111}$ In-Antimyosin antibody imaging, and ${ }^{99 \mathrm{~m}} \mathrm{Tc}$-Annexin $\mathrm{V}$ apoptosis imaging, biomarkers including troponins and BNP; genetic markers, and both functional and tissue characterization techniques with T1 weighted and T2 weighted images with cardiac magnetic resonance imaging (CMR). In our quest to optimize strategies for long-term cancer survival and prevention of CHF for patients receiving chemotherapy, rigorous modality and guideline-specific clinical outcome trials are required. A new multi-modality monitoring approach is proposed, which integrates evidencebased strengths of CMR, echocardiography, ERNA, biomarkers, and BP management for surveillance and validation of cardiotoxicity and prevention of clinical heart failure in patients receiving a broad spectrum of cancer therapies.

Key Words: Radionuclide angiography $\bullet$ magnetic resonance imaging $\bullet$ echocardiography $•$ cardiomyopathy $\cdot$ heart failure

Primum non nocere

- Author unknown
From the University of Rochester Medical Center, ${ }^{a}$ Rochester, NY; and Westchester Medical Center, ${ }^{\text {b }}$ New York Medical College, Valhalla, NY.

Reprint requests: Ronald G. Schwartz, MD, MS, FACC, FAHA, ABNM, FASNC, University of Rochester Medical Center, Rochester, NY; ronald_schwartz@urmc.rochester.edu.

J Nucl Cardiol 2013;20:443-64.

$1071-3581 / \$ 34.00$

Copyright (c) 2013 American Society of Nuclear Cardiology.

doi:10.1007/s12350-013-9707-1

\section{INTRODUCTION}

Cancer patients receiving chemotherapy have an increased risk of developing cardiotoxicity, and this risk is enhanced with a prior history of heart disease. Anthracycline-induced cardiotoxicity can be categorized as acute, early-onset chronic progressive, and late-onset chronic progressive. ${ }^{1}$ Acute cardiotoxicity occurs in $<1 \%$ of patients immediately after anthracycline administration and results in acute, transient decline in myocardial contractility, and/or self-limited dysrhythmias. The earlyonset chronic progressive form occurs in $1.6-2.1 \%$ of patients, during therapy or within the first year after treatment. ${ }^{1}$ Late-onset chronic progressive anthracyclineinduced cardiotoxicity occurs at least 1 year after 
completion of therapy in $1.6-5 \%$ of patients. ${ }^{1}$ Serious complications of chemotherapy include transient, early- or late-onset left ventricular dysfunction and congestive heart failure (CHF). Late-onset cardiotoxicity may be noted as long as 20 years after the first dose of cancer treatment, ${ }^{1}$ and confounding myocardial insults associated with hypertension, coronary heart disease, valvular disease, thromboembolism, and radiation may augment risk of cardiac dysfunction and CHF. The spectrum of chemotherapy-induced cardiotoxicity includes hypertension, vasospasm causing angina or myocardial infarction, dysrhythmias, bradycardia, QT prolongation with rare but potentially life threatening torsade de pointes, and right heart failure and diastolic dysfunction associated with pulmonary fibrosis. ${ }^{1-4}$ Cardiopulmonary insufficiency due to $\mathrm{CHF}$, pulmonary dysfunction or both may cause serious morbidity and death. Lefrak et $\mathrm{al}^{5}$ first reported four decades ago deaths due to CHF as early as two weeks following the final dose of doxorubicin. Since then, a widespread implementation of serial assessment of resting left ventricular ejection fraction (LVEF) with equilibrium radionuclide angiocardiography and echocardiography ${ }^{6-15}$ in adults and children, and modification or discontinuation of chemotherapy at the appearance of subclinical LV dysfunction or characteristic histopathologic changes on endomyocardial biopsy ${ }^{16}$ prior to the development of CHF have fundamentally improved its natural history.

A complex landscape of therapeutic options and diagnostic methods to prevent chemotherapy-induced CHF confronts the cardio-oncologist in the current era. Adverse cardiovascular effects of anticancer agents are summarized in Table $1 .{ }^{15}$ While endomyocardial biopsy can detect progressive, cumulative dose-related increases in histopathological changes,${ }^{16}$ empiric dose restrictions designed to limit CHF in populations receiving chemotherapy ${ }^{17}$ fail to address the large inter-individual variation of the cumulative dose that precipitates CHF. ${ }^{8,9}$ Successful management of LV dysfunction and CHF prevention were based on concordance with specific guidelines in populations predominantly dosed every 3-4 weeks with $50-75 \mathrm{mg} / \mathrm{m}^{2}$ doxorubicin. ${ }^{8}$ Greater dose intensity of anthracycline therapy can now be employed as marrow reserves are protected by colony stimulating factors and marrow transplantation. Many additional chemotherapeutic agents with differing mechanisms of therapeutic effectiveness and cardiotoxicity compound the challenges of safe chemotherapy management (Table 2). Cancer patients cured of their malignancies are living longer, increasing the chance of developing late-onset cardiomyopathy and $\mathrm{CHF}{ }^{15}$

Safety of higher dose intensity therapy, treatment of the extremes of age most sensitive to cardiotoxicity of chemotherapy, and the optimal method and cut points for optimizing chemotherapeutic effectiveness while
Table 1. Toxicity of chemotherapeutic agents

\begin{tabular}{|c|c|}
\hline Agent & $\begin{array}{c}\text { Most frequent } \\
\text { toxicity }\end{array}$ \\
\hline Fluoracil & $\begin{array}{l}\text { Myocardial ischemia } \\
\text { and infarction }\end{array}$ \\
\hline Anthracyclines & $\begin{array}{l}\text { Cardiomyopathy, } \\
\text { myopericarditis, } \\
\text { arrhythmias }\end{array}$ \\
\hline Cisplatin & Hypertension \\
\hline Cyclophosphamide & $\begin{array}{l}\text { Heart failure, } \\
\text { myopericarditis, } \\
\text { arrhythmias }\end{array}$ \\
\hline Taxanes & $\begin{array}{l}\text { Heart failure, ischemia, } \\
\text { arrhythmias }\end{array}$ \\
\hline Methotrexate & Ischemia, arrhythmias \\
\hline Trastuzumab & Heart failure \\
\hline Tamoxifen & Venous thrombosis \\
\hline Radiotherapy & $\begin{array}{l}\text { Restrictive heart } \\
\text { disease, accelerated } \\
\text { atherosclerosis, } \\
\text { pericardial effusion }\end{array}$ \\
\hline
\end{tabular}

Source Ref. 15.

preventing or limiting CHF require careful study of evidence-based outcomes. Noninvasive methods that measure LVEF may not have adequate accuracy or precision to detect real changes on serial evaluation and prevent CHF effectively. Without carefully collected outcome data, it remains unclear whether addition of invasive or noninvasive evidence of histological cardiotoxicity provides incremental value of optimizing chemotherapeutic effectiveness while preventing CHF. The purpose of this article is to review the evidence base of effectiveness of monitoring cardiac dysfunction and preventing CHF in adults and children with traditional and novel noninvasive methods, including biomarkers, and to consider promising approaches for further investigation in the field. A new multi-modality monitoring approach is proposed, which integrates evidence-based strengths of cardiac magnetic resonance imaging (CMR), echocardiography, ERNA, biomarkers, and blood pressure (BP) management for surveillance and validation of cardiotoxicity and prevention of clinical heart failure in patients receiving a broad spectrum of cancer therapies.

\section{HYPERTENSION}

Chemotherapy-induced hypertension is a common adverse effect of angiogenesis inhibitors and immunosuppressants and demonstrates the need for routine BP 
Table 2. Chemotherapeutic drugs with cardiotoxic effects

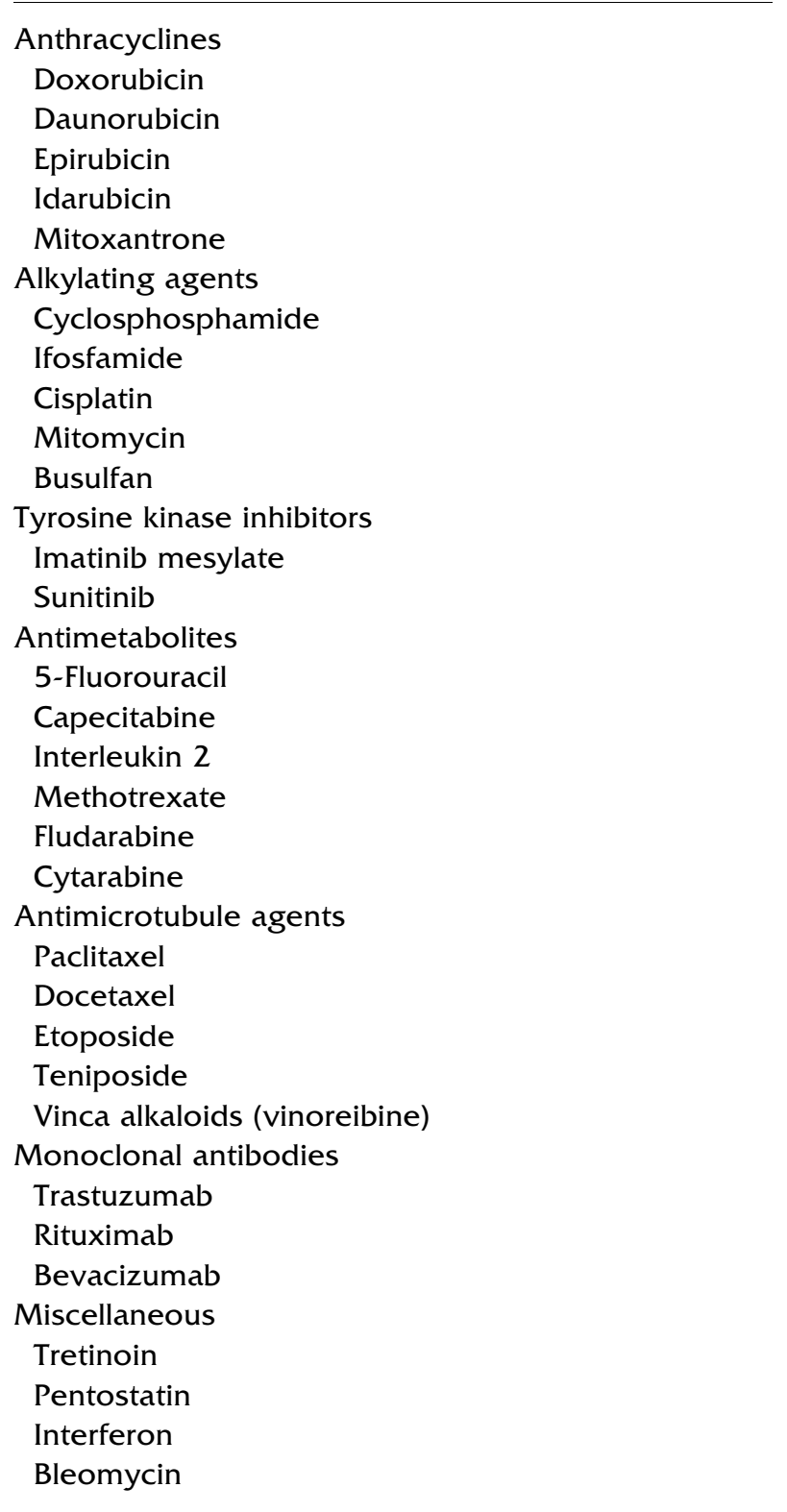

monitoring and guideline-based management of hypertension. ${ }^{3}$ Recent recommendations ${ }^{4}$ for management of hypertension include: (1) Baseline BP measurement so pre-existing hypertension, which is common in cancer patients, can be reliably identified and treated before initiation of vascular signaling pathway (VSP) inhibitors (e.g., anti VEGF antibody bevacizumab, and certain tyrosine kinase inhibitors). (2) Active monitoring of BP throughout the period of cancer management, especially during the initiation of chemotherapy when most patients experience secondary elevation in BP. (3) Application of
JNC guidelines to manage $\mathrm{BP}$, including target $\mathrm{BP}$ below $140 / 90 \mathrm{mmHg}$ in general and below $130 / 80 \mathrm{mmHg}$ in patients with diabetes mellitus or kidney disease. (4) Aggressive BP control to minimize the risk of end-organ damage. (5) Attention to the choice of antihypertensive medication is also recommended. Special consideration for the use of ACE inhibitors or angiotensin receptor blockade therapy and carvedilol is warranted because of the demonstrated cardioprotective effects of these agents in this patient population. The referral of patients to hypertension specialists is recommended whenever oncologists face difficulties in achieving adequate BP control. Thus, BP measurement is a traditional method of avoiding acute and downstream complications of hypertension-induced systolic and diastolic HF in patients receiving cancer chemotherapy, particularly VSP inhibitors, and merits consideration within the scope of this review.

\section{CLINICAL MANIFESTATIONS OF ANTHRACYCLINE CARDIOTOXICITY}

Anthracyclines are highly effective, broad-spectrum anti-neoplastic agents, and cardiotoxicity is a major limitation of their administration. Anthracyclines were originally isolated from the bacteria Streptomyces peucetius and utilized as antibiotics. They act by inhibiting DNA and RNA synthesis by intercalating between base pairs and by inhibiting the activity of topoisomerase II which prevents DNA repair. Acute and chronic stages of cardiotoxicity with anthracycline therapy have been described. Acute onset toxicity may occur during or soon after initiation of therapy and can be associated with non-diagnostic repolarization changes, dysrhythmias, a pericarditis-myocarditis syndrome with troponin elevations, and transient ventricular dysfunction. These manifestations are considered uncommon, do not persist, and do not require routine monitoring. Chronic cardiotoxicity is the more common manifestation which usually presents as symptomatic LV dysfunction associated with a dilated cardiomyopathy. Chronic toxicity may be considered Type 1 or early-onset and Type 2 or lateonset chronic progressive phase. This chronic cardiotoxicity associated with progressive increases in total cumulative doses of anthracycline therapy is insidious, associated with progressive myocellular loss due to apoptosis and necrosis, ${ }^{15,16}$ and causes LV remodeling which results in asymptomatic LV dysfunction which precedes symptomatic CHF. ${ }^{6-9,15}$ Chronic anthracycline cardiotoxicity can present as a restrictive cardiomyopathy with diastolic dysfunction which may be worsened by radiation-induced fibrosis, but more typically presents as dilated cardiomyopathy with systolic dysfunction. Prior to widespread use of noninvasive monitoring, the 
incidence of CHF was more than $4 \%$ in patients receiving $500-550 \mathrm{mg} / \mathrm{m}^{2}, 18 \%$ in those receiving cumulative dosages of $551-600 \mathrm{mg} / \mathrm{m}^{2}$, and to $36 \%$ at doses of doxorubicin over $601 \mathrm{mg} / \mathrm{m}^{2} ., 17$ In this pre-LV monitoring era of the 1970s, the incidence of CHF peaked at 3 months after last anthracycline dose and mortality of these patients was $60 \% .^{5,17}$

The relationship of total cumulative dose of anthracycline therapy with linearly progressive histopathology of myofibrillar disarray and apoptosis on endomyocardial biopsy and progressive increase in CHF in populations studied lead to recommendations to limit empirically the dose of doxorubicin administered. ${ }^{17}$ The major problem with empiric dose limitation is the large inter-individual variation of total cumulative dose of doxorubicin that precipitates CHF, and some patients may develop clinical CHF at relatively low doses of doxorubicin. ${ }^{8,9}$ Furthermore, CHF can be safely prevented during doxorubicin therapy in patients with baseline LV dysfunction provided guidelines for frequent serial measurements of LVEF and termination of chemotherapy are followed. ${ }^{7,8}$ Although monitoring quantitative LVEF limits the prevalence and severity of $\mathrm{CHF}$ and permits effective treatment of CHF when it occurs, monitoring cardiac dysfunction has not entirely eliminated $\mathrm{CHF}$ in anthracycline-treated patients.

Doxorubicin results in progressive myocytolysis and sarcomere destruction and development of restrictive and dilated cardiomyopathy. Despite extensive studies in animals and cell culture models, the exact cellular, biochemical, molecular, and genetic mechanisms of cardiotoxicity associated with anthracycline and other chemotherapies are not fully understood. Extremes of age, underlying cardiac risk factors (hypertension, hyperlipidemia, family history, diabetes, smoking), concomitant treatment with radiation therapy, high-dose cyclophosphamide, and pre-existing heart disease have been identified as predisposing factors for doxorubicin cardiotoxicity. Other classes of chemotherapeutic agents may potentiate anthracycline toxicity, or have their own cardiotoxic effects. Chemotherapeutic agents with cardiotoxic effects are listed in Tables 1 and 2 and include, in addition to anthracyclines, alkylating agents, tyrosine kinase inhibitors, antimetabolites, antimicrotubule agents, monoclonal antibodies such as trastuzumab, ${ }^{18,19}$ and miscellaneous agents such as interferon and bleomycin.

Endomyocardial biopsy directly measures the severity of cumulative dose related anthracycline induced histopathology. Limitations of this technique include invasiveness, sampling limitations, requirement for specialized expertise to interpret the results, and moderately higher expense. ${ }^{20}$ Guidelines for use of myocardial biopsy in the management of heart failure have been published. ${ }^{21}$ In the current era, endomyocardial biopsy is not used for serial monitoring of chemotherapy-induced cardiotoxicity. Endomyocardial biopsy is utilized to clarify diagnostic evaluation of the etiology of new onset CHF. Although empiric dose limitations have been advocated to reduce the risk of CHF with anthracycline therapy ${ }^{17}$ large inter-individual variation in the tolerance to total cumulative dose of anthracyclines and differences in the mechanisms and risks of cardiotoxicity of newer agents suggest the value of a review of traditional and novel methods of monitoring cardiotoxicity and cardiac dysfunction to maximize therapeutic value and safety of cancer chemotherapy, as noted in Table 3. This review will summarize our current understanding of the noninvasive monitoring methods and propose potentially fruitful areas for further basic and clinical investigation in this field. Finally, a new multimodality integrative approach to monitoring chemotherapyinduced LV dysfunction is proposed.

Table 3. Traditional and novel methods to assess chemotherapy-related cardiac dysfunction noninvasively

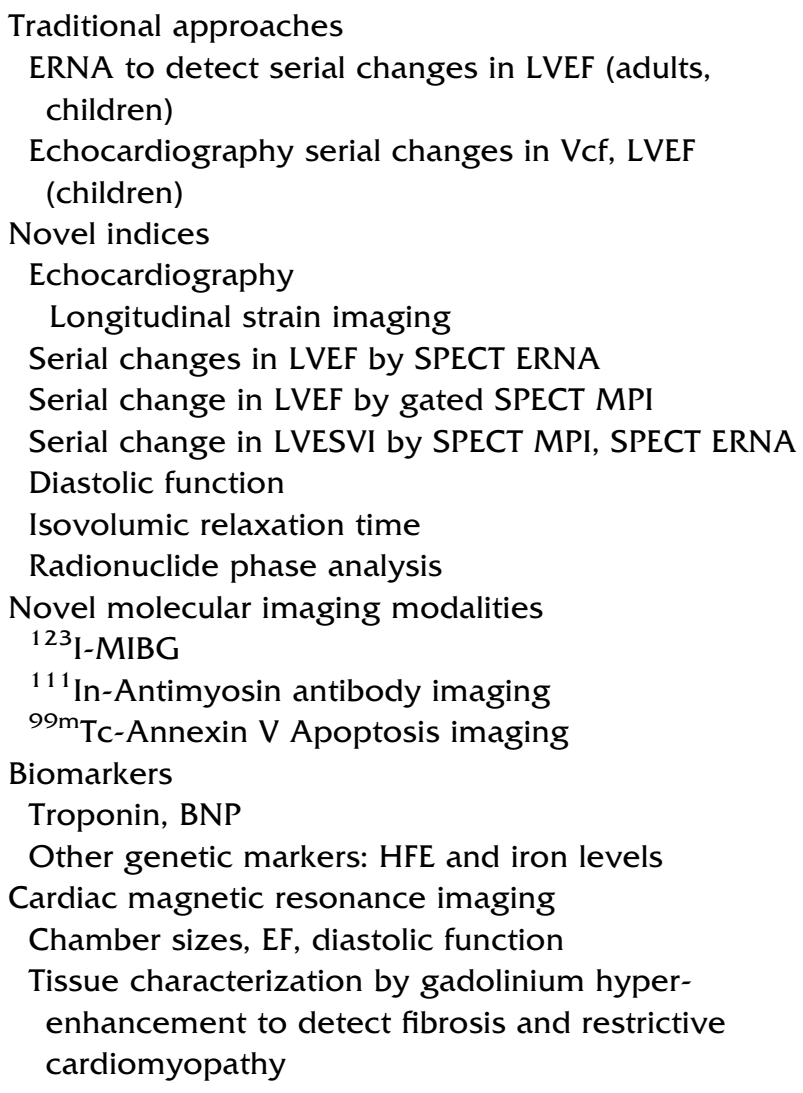




\section{IMAGING MODALITIES}

\section{ERNA Monitoring of Anthracycline Cardiotoxicity}

Following the development of endomyocardial biopsy technique by Billingham et al, ${ }^{16}$ which showed a linear progression of histopathological myocardial changes with increasing cumulative dosages of anthracycline therapy, Alexander et $\mathrm{al}^{6}$ pioneered the concept of predicting doxorubicin-induced CHF by monitoring change in resting LVEF using ERNA. Patients who progressed to overt $\mathrm{CHF}$ had a decline in LVEF below the lower limit of normal prior to onset of $\mathrm{CHF}^{6} \mathrm{Choi} \mathrm{et} \mathrm{al}^{7}$ demonstrated patients with abnormal resting LVEF by ERNA could safely receive doxorubicin provided LVEF remained above $30 \%$ prior to each dose, and the decline in EF was less than $10 \mathrm{EF}$ units. From a compiled registry of 1,487 patients over seven years who underwent serial radionuclide monitoring at university and community hospitals, Schwartz et $\mathrm{al}^{8}$ correlated the observation of changes in LVEF by ERNA and CHF outcome in 282 high risk patients. These high risk patients were selected for evaluation for $\mathrm{CHF}$ outcome based on either high total cumulative dose of doxorubicin $\left(>450 \mathrm{mg} / \mathrm{m}^{2}\right)$, decline in LVEF by at least $10 \mathrm{EF}$ units to LVEF $50 \%$ or less, and/ or abnormal baseline LVEF $<50 \%$. CHF was noted in $46(16 \%)$ patients during the treatment period and an additional 3 patients $(1.3 \%)$ on 1 year post therapy follow-up. Total cumulative dose which precipitated CHF $\left(75-1,095 \mathrm{mg} / \mathrm{m}^{2}\right)$ and the dose that did not (30$880 \mathrm{mg} / \mathrm{m}^{2}$ ) varied widely. CHF was noted mostly in patients with normal baseline LVEF that declined by $10 \%$ to a value of $<50 \%$. CHF was treatable and improved in $87 \%$ of patients given digitalis, diuretic and/or vasodilator therapy. No death was attributed to CHF in this cohort.

Guidelines for monitoring patients receiving doxorubicin therapy and avoiding CHF based on the analysis of CHF outcomes in this high risk group of patients are listed in Table 4 and continue to guide anthracycline therapy in the current era. The guidelines recommend a baseline ERNA measurement of LVEF. Subsequent studies are performed 3 weeks after the last dose (or just before the administration of next planned dose). For patients with a normal baseline LVEF, the second ERNA is performed at $250-300 \mathrm{mg} / \mathrm{m}^{2}$. The next ERNA at $450 \mathrm{mg} / \mathrm{m}^{2}$ unless risk factors such as prior or concomitant cyclophosphamide therapy, heart disease, mediastinal radiation, or abnormal ECG are present in which case the next study is performed at $400 \mathrm{mg} / \mathrm{m}^{2}$. Discontinuation of doxorubicin is recommended if LVEF decreases $\geq 10 \%$ (EF units) from baseline and reaches LVEF $\leq 50 \%$. For patients with abnormal baseline LVEF $<50 \%$, serial studies are recommended after each
Table 4. Guidelines for serial monitoring of LVEF by ERNA

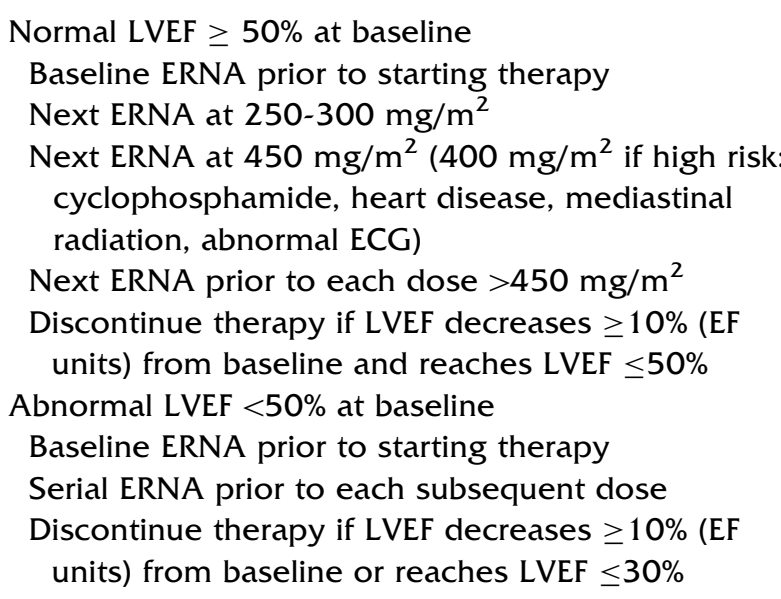

Serial studies are performed at least 3 weeks after the last dose.

Source Ref. 8.

dose of doxorubicin. Discontinuation of doxorubicin is recommended if LVEF decreases $\geq 10 \%$ (absolute EF units) from baseline OR reaches LVEF $\leq 30 \%{ }^{8}$

Concordance in management with the specific parameters of these guidelines was assessed relative to CHF outcome in this study. Those patients whose management was not strictly concordant with these guidelines despite serial monitoring of quantitative LVEF had significantly higher incidence of CHF as illustrated in Figure 1. Severity of CHF was also higher in those patients not managed in accordance with the recommended guidelines. In summary, monitoring resting LVEF with serial ERNA is associated with a low incidence, benign course, and reversible degree of CHF. In a population at high risk of developing anthracycline cardiotoxicity, who were monitored with serial ERNA, strict adherence to the specific guidelines for timing of studies and termination of chemotherapy which were developed over seven years and subsequently validated in clinical practice over three decades, reduces the incidence and severity of CHF. These observations were extended by Mitani et $\mathrm{al}^{9}$ who showed serial changes in EF by ERNA identified risk of CHF in patient cohorts receiving similar average cumulative doses of doxorubicin with wide dose variation, including some patients with relatively low dose. This observation again supports the concept that serial monitoring by highly accurate serial radionuclide LVEF and adherence to specific treatment guidelines starting early in the course of chemotherapy is effective to prevent or limit CHF. In addition, the cost effectiveness analysis showed the total financial cost of serial ERNA studies by recommended guidelines was 


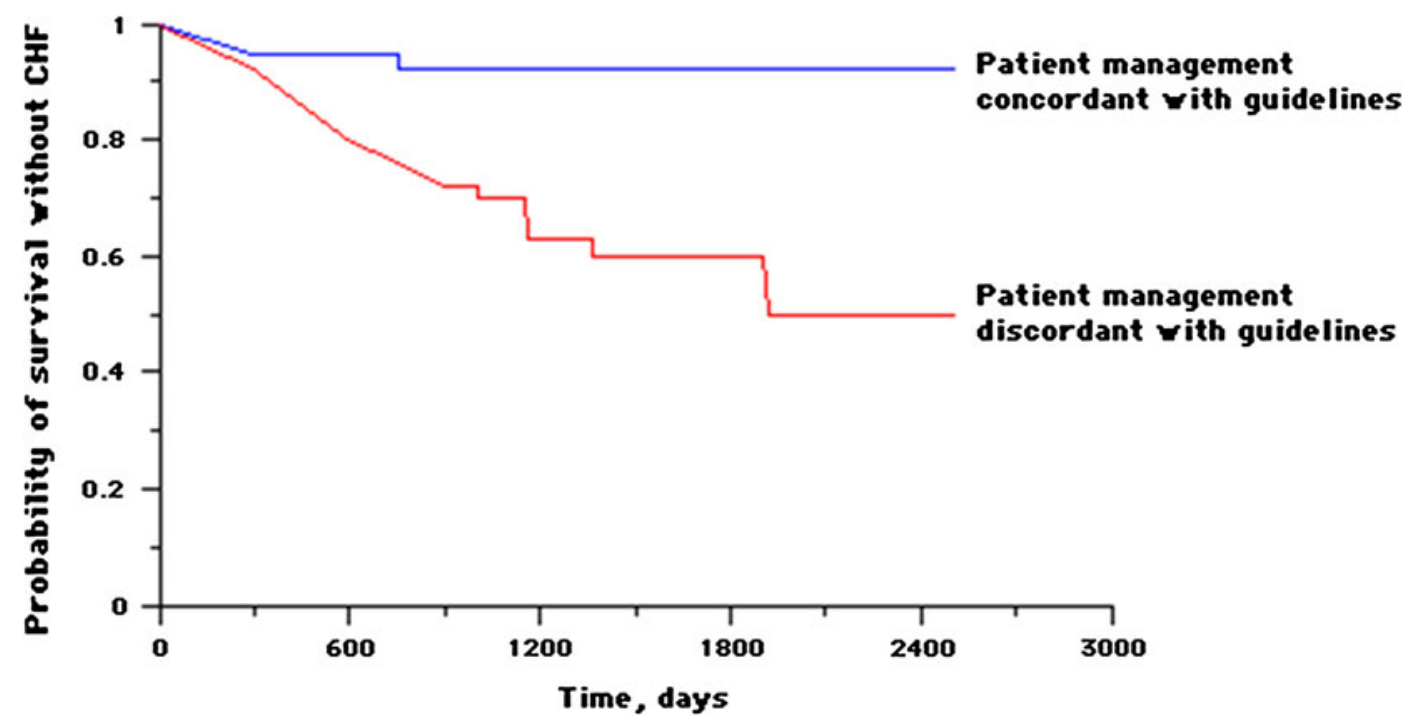

Figure 1. Guidelines prevent doxorubicin cardiotoxicity. Kaplan-Meier plot describes the probability of survival without clinical CHF in patients whose management was either concordant or discordant with the guideline criteria for monitoring patients receiving doxorubicin. Patients managed by the guidelines had a fourfold reduction in the incidence of clinical CHF independent of other predictor variables $(P<.01)$. When CHF did occur with guideline concordant management, severity of CHF was no worse than mild. By comparison, the severity of clinical CHF was worse than mild in the majority in the group of patients whose management was discordant with the guidelines. Source Ref. 8.

lower than the 1-year cost of caring for additional cases of CHF that would be expected without the preventive benefit of routine accurate ERNA LVEF monitoring. ${ }^{9}$

Thus, serial resting ERNA performed by specific guidelines during doxorubicin therapy reliably monitors cardiotoxicity and identifies patients who safely tolerate high cumulative doses of doxorubicin. ERNA offers advantages of high accuracy and reproducibility with lower inter-observer variability $(<5 \%)$, greater reliability and outcomes based clinical validation than has been demonstrated to date by 2D echocardiography in adults. Exposure to radiation is frequently and uncritically cited as a disadvantage of ERNA. However, its appropriate guideline directed utilization and proven clinical value for preventing CHF far outweigh the unmeasurably low theoretical cancer risk of exposure to low dose radiation of nuclear cardiology studies. In 2013, studies are required to validate the effectiveness of serial LVEF monitoring for anthracycline cardiotoxicity in high risk patients of other techniques such as gated SPECT myocardial perfusion imaging, echocardiography with and without contrast, and CMR. Beyond correlation of LVEF and LV volume indices, prospective validation in high risk patient populations is warranted to optimize safe administration of chemotherapy and to demonstrate management concordant with chemotherapy treatment guidelines influences CHF risk, morbidity, and response to CHF therapy and cost effectiveness as has been demonstrated with ERNA. ${ }^{6-9}$

\section{Modifiers for ERNA Follow-Up}

Age greater than 65, hypertension, use of alkylating agents like cyclophosphamide, known heart disease, and mediastinal radiation therapy have been considered risk modifiers for anthracycline-induced cardiotoxicity which warrant more frequent LVEF assessment (at 400 instead of $450 \mathrm{mg} / \mathrm{m}^{2}$ ). ${ }^{8}$ Iron overload due to transfusions, nutrition, and genetic mutations of iron handling may also warrant earlier and more frequent evaluations of LVEF based on data of enhanced doxorubicin cardiotoxicity in iron-loaded rodents. ${ }^{22}$ The mitigating effects of slower infusion rate and liposomal doxorubicin also warrant further study.

\section{Non-anthracycline Chemotherapy Cardiotoxicity}

Trastuzumab (Herceptin) is another cancer therapeutic agent with a well-defined and predictable risk of cardiotoxicity. However, the cardiotoxicity and cardiac dysfunction of trastuzumab tends to be treatable and fully reversible within a few months upon discontinuation of therapy, and is not associated with discernible ultrastructural changes and is non-cumulative. ${ }^{18}$ Ewer et al reported in 38 patients with HER2/neu-positive breast cancer referred for trastuzumab-induced cardiotoxicity a decline of mean LVEF from $61 \%$ to $43 \%$ 
which increased to $56 \%$ at a mean time to recovery of LVEF of 1.5 months. All patients in this observational series had previously received anthracycline chemotherapy. This improvement of LVEF was temporally associated with medical treatment of CHF in $32(84 \%)$ of the 38 patients and occurred without treatment in six patients $(16 \%)$. Twenty two of 25 patients $(88 \%)$ who were re-challenged with trastuzumab, all of whom were treated medically for CHF, did not develop recurrent LV dysfunction. Thus, re-introduction of trastuzumab may be appropriate for some patients who previously experienced trastuzumab-related cardiac dysfunction. ${ }^{18}$ Trastuzumab-induced cardiomyopathy is self-limiting in the absence of prior anthracycline exposure, and rechallenging with trastuzumab is often well tolerated without further decline in LVEF, unlike doxorubicininduced LV dysfunction. ${ }^{18,19}$ The effect of trastuzumab on patients with prior cardiac disease remains unclear, and it is recommended such patients be very closely monitored. ${ }^{18,19,23}$ In the absence of large prospective clinical trials, alternative algorithms for management during adjuvant trastuzumab therapy have been published by Panjrath and Jain (Figure $2^{19}$ ) and the Canadian Trastuzumab Working Group (Table $5^{23}$ ).

Cardiotoxicity has been reported in up to $2.3 \%$ of patients receiving 5-fluorouracil. ${ }^{24}$ Coronary vasospasm associated with 5-fluorouracil is associated with ischemia, chest pain, ECG repolarization changes, myocardial infarction, and death. Prospective study of 100 consecutive patients without cardiac history or abnormal ECG at baseline showed 8 patients $(8 \%)$ developed chest pain, ECG changes, and one case of cardiogenic shock within 18-30 hours of the initiation of the high-dose 5-fluorouracil infusion. ${ }^{25}$ These adverse effects were reportedly not associated with biomarker release, and the symptoms resolved with discontinuation of the 5-fluorouracil. ${ }^{25}$ These findings suggest the benefit of ECG monitoring of patients receiving 5-fluorouracil, particularly high-dose therapy.

\section{Other Radionuclide Ventriculographic Approaches: Exercise RNA, Resting ECG- Gated SPECT ERNA, and MPI}

The extensive evidence base of effectiveness in safe monitoring of anthracycline therapy has been based on serial measurements of planar ERNA at rest. ${ }^{6-9}$ Baseline decline of LVEF from rest to exercise by RNA in 48 patients reported by Palmeri et $\mathrm{al}^{26}$ appeared to enhance detection of cardiotoxicity but did not enhance detection of CHF compared to age and mid-course resting LVEF. Exercise ERNA in the current era lacks relevance in clinical oncology for several reasons: only very limited data from very small studies are available, most labs currently are not well equipped to perform exercise ERNA, and the oncology patient populations is much

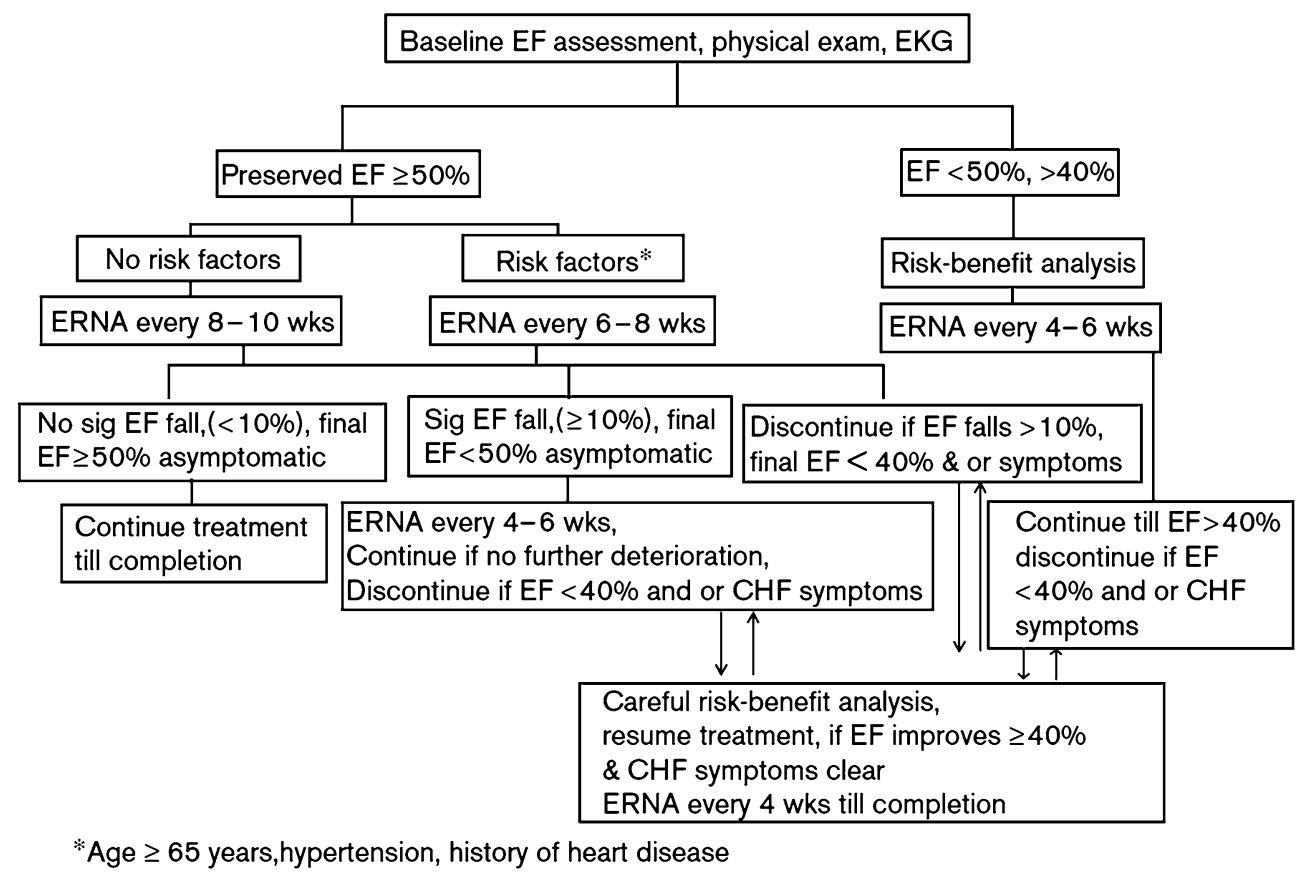

A proposed algorithm for monitoring trastuzumab cardiotoxicity.

Figure 2. A proposed algorithm for monitoring trastuzumab cardiotoxicity. Source Ref. 19. 
Table 5. Considerations for monitoring and management of trastuzumab-induced cardiotoxicity

Assessment of cardiac function per established protocols is critical and must be endorsed for all patients

Either echocardiography or multiple-gated acquisition scan should be used to establish baseline LVEF. The same imaging modality should be used at follow-up

Multiple-gated acquisition scanning is generally more widely available in Canada and may be subject to less variability

If echocardiography is used, the same technique must be used for each assessment. The preferred technique is the Simpson method

The LVEF should be assessed before trastuzumab treatment is started (and after chemotherapy, for sequential regimens) and should be repeated every 3 months until completion of trastuzumab therapy. Each patient will therefore undergo a minimum of 5 LVEF assessments: immediately before trastuzumab is initiated and at 3, 6, 9 , and 12 months in the course of therapy

Patients who experience cardiac symptoms or a greater than $10 \%$ absolute asymptomatic decline in LVEF while receiving trastuzumab may continue to undergo annual cardiac assessments following completion of trastuzumab treatment

At this time, no evidence exists to support further cardiac monitoring of patients who have completed chemotherapy and trastuzumab treatment with no cardiac symptoms and no signs of substantial (greater than $10 \%$ absolute decrease), but asymptomatic, LVEF decline

The cardiac monitoring requirements outlined in this article should be understood to represent the minimum monitoring requirements. Patients with cardiotoxicity or other risk factors may require more frequent and more stringent monitoring

Source Ref. 23.

sicker and older now compared to three decades ago. Thus, a substantial majority of oncology patients would not be able to perform adequate exercise. For these reasons, exercise RNA is not currently recommended for evaluation of cancer patients.

All studies for monitoring cardiotoxicity of cancer chemotherapy have been carried out using planar ERNA. However, recent availability of ECG-gated SPECT ERNA offers advantages over the conventional planar ERNA in monitoring chemotherapy-induced cardiotoxicity and risk of $\mathrm{CHF} .{ }^{27}$ The accuracy of gated blood pool SPECT (GBPS) software for the calculation of LVEF has been validated. ${ }^{27}$ The software provides sophisticated wall motion and phase analyses in addition to right ventricular ejection fraction (RVEF), LVEF, and left and right ventricular end-systolic and end-diastolic absolute volumes. End-systolic volume index (ESVI) is a powerful marker of risk in patients with known or suspected coronary heart disease, ${ }^{28,29}$ and may also be a useful parameter to quantify risk of CHF in patients receiving cancer chemotherapy. A gender difference with worse outcome for women compared to men has been found with progressive reduction of LVEF and increases in ESVI and EDVI in women with similar pretest risk of ischemic heart disease, ${ }^{28}$ but the assessment of gender difference in risk of CHF with cancer chemotherapy has not been reported to date. A high degree of accuracy and precision of LVEF measurements by SPECT ERNA ${ }^{15,30}$ suggest its interchangeability with planar ERNA for monitoring risk of $\mathrm{CHF}$ in patients receiving cancer chemotherapy. However, the incremental value of RVEF, LVESVI, and LVEDVI provided by SPECT ERNA over and above the conventional LVEF monitoring in predicting and preventing CHF in cancer population is unknown and remains an important opportunity for further research. LVEF, LVESVI, and EDVI can also be derived from gated SPECT myocardial perfusion imaging, and their gender-specific prognostic value in patients referred for ischemia evaluation has been reported. ${ }^{28}$ However, a number of methodological variables have been noted ${ }^{31}$ and it is unclear if this technique offers any advantages over the LVEF assessment by planar or SPECT ERNA in cancer patients, unless simultaneous information about myocardial perfusion is required for pre-existing or suspected coronary artery disease. ${ }^{32,33}$

Radionuclide evidence of differences in diastolic peak filling rate by epirubicin and doxorubicin were reported. ${ }^{34}$ Although reduced diastolic peak filling rates enhance sensitivity for identifying early anthracycline cardiotoxicity, to date no evidence has identified its ability to improve prediction of clinical CHF compared to serial assessment by ERNA of LVEF during chemotherapy. ${ }^{6-9}$ Multicenter assessment of the interoperator reproducibility of serial LVEF measures by ERNA is reported to be within $3.2 \%$ (EF units) for planar imaging compared to $4.1 \%$ for SPECT imaging. Calculated LVEF by GBPS was $8 \%$ higher than by planar analysis, owing to the exclusion of the left atrium. ${ }^{35}$ 


\section{ECHOCARDIOGRAPHY FOR ASSESSMENT OF LVEF, LVESVI AND RISK OF CHF}

Echocardiography is often used to monitor LVEF and LV volumes in clinical cardiology. Advantages of echocardiography include its wide availability and lower test cost. Disadvantages include limitations of accuracy associated with geometric assumptions of chamber shape, acoustic artifact limiting window size, operator-dependent assessment, subjective analysis, and predominantly $2 \mathrm{D}$ analysis. The $95 \%$ confidence intervals of measured LVEF by $2 \mathrm{D}$ echocardiography are $\pm 11 \%$, a wide variation which limits accuracy and precision of LVEF assessment which can cause failure to detect significant changes in LVEF. ${ }^{15}$ The value of echocardiography for monitoring cardiotoxicity, managing chemotherapy, and preventing $\mathrm{CHF}$ in high risk adult patient populations receiving cancer chemotherapy has not been critically evaluated in a prospective and independent manner. A presumed role of echocardiographic assessment of LVEF in monitoring the risk of CHF in cancer patient populations evolved predominantly as a result of the extrapolation from the role of quantitative ERNA which has been proven to prevent $\mathrm{CHF}$ in adults. However, such extrapolations may be limited by the differences in the objectivity, accuracy, and reproducibility of the LVEF measurements by echocardiographic and nuclear imaging techniques. ${ }^{15}$ The role of ESVI by echocardiography as a predictor of CHF hospitalization in stable CAD have been reported in the Heart and Soul Study. ${ }^{36}$ In contrast to the adult patient population, echocardiography with or without ERNA has become a standard for evaluation in pediatric patient population, ${ }^{10-14}$ where smaller patient size permits effective use of higher frequency transducers, with better image quality and higher spatial resolution, and less acoustic artifact than in adults (Figure 3). The use of contrast represents an important opportunity for further investigation in children and adults treated with chemotherapy, as the American Society of Echocardiography guidelines state the underestimation of cardiac volumes by echocardiography can be nearly resolved when contrast agents are used. ${ }^{15,37}$

Very recently, the role of contrast use in echocardiography to optimize accuracy and precision of serial EF and LV volume measurements by traditional echocardiography has been addressed in adults. ${ }^{38}$ To be able to detect a $5 \%$ change in EF with confidence, the measurement technique should have inter-measurement variability of less than the sum of its reported mean plus its $2 \mathrm{SD}$ at $5 \%$, i.e., the upper limit of the confidence interval needs to be $5 \%$ to guarantee that, in $90 \%$ of the patients in whom a decrease $5 \%$ in LVEF is detected, this decrease would indeed be a meaningful finding and not a

\section{Guidelines for Monitoring Anthracycline Cardiotoxicity in Children}

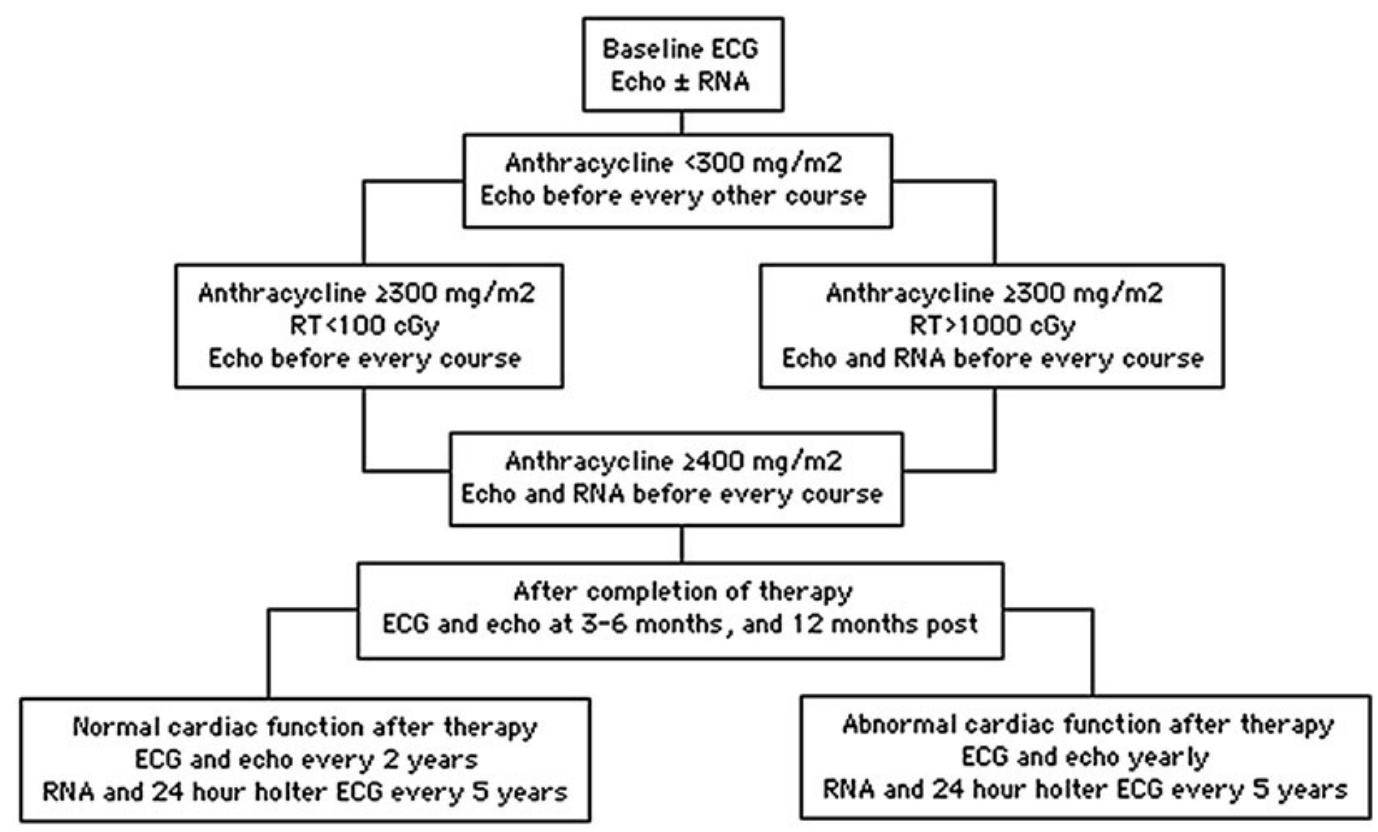

$E C G=$ electrocardiogram; $E c h o=$ echocardiogram; RNA = radionuclide angiocardiogram; RT = radiation therapy. Adapted from Steinherz, LJ, Graham, T, Hurwitz, R, et al, Pediatrics 1992; 89:942.

Figure 3. Guidelines for monitoring anthracycline cardiotoxicity in children. 
measurement error. ${ }^{39}$ Among six echocardiographic techniques tested for the measurement of the three parameters (end-systolic- and end-diastolic volume and EF), the 3D measurement of EF provided sufficient reproducibilityas reflected by the upper limit of the confidence interval, which is $4.9 \%$ (i.e., just below the 5\% target) to suggest its appropriate use for serial EF monitoring to assess a 5\% change. Importantly, all four 2D techniques showed temporal variability that was roughly twice as high. 3D echocardiography (3DE) has been reported in a study of 56 patients to optimize the reproducibility of serial measurements of LVEF in patient with stable function over a one-year period of time and was found to be the most reliable echocardiographic method for determination of LVEF. ${ }^{38}$ The accompanying editorial suggests the reproducibility of 3DE in this single center study appears potentially sufficient to provide reliable measurement of clinically meaningful changes in LVEF. However, the accompanying editorial cautions future multicenter studies will be required to determine if these findings can be extrapolated to the general population of cancer patients receiving chemotherapy, ${ }^{39}$ which remains unknown. Thus, in 2013, it remains to be demonstrated if reliable guidelines can be developed to inform management decisions of suspension of anthracycline therapy to prevent LV dysfunction and heart failure in high risk patients while optimizing chemotherapeutic effectiveness using echocardiographic measurements of LVEF.

While the accuracy and precision of serial LVEF measurements by echocardiography require further evidence for reliability of clinical application for monitoring chemotherapy, other echocardiographic indices of longitudinal strain, tissue Doppler imaging, isovolumic relaxation indices, left atrial size are promising candidates for detection of subclinical cardiotoxicity. Echocardiographic evidence that LV diastolic dysfunction precedes resting systolic dysfunction has been reported, but does not appear to correlate with doxorubicin dosage or enhance prediction of $\mathrm{CHF}^{40-42}$ These parameters include prolonged isovolumetric relaxation period, reduction in peak flow velocity, and the ratio of early peak flow velocity/atrial peak flow velocity, as well as reduction in the deceleration rate of the early peak flow velocity. Stoddard et $\mathrm{al}^{41}$ reported prolongation of isovolumetric relaxation time (IVRT) by Doppler echocardiography to predict doxorubicin-induced systolic dysfunction in a study of 26 patients. Doxorubicin-induced decline of EF by $>10-55 \%$ or less was noted in 9 of 26 patients. Isovolumetric relaxation time was prolonged from $66 \pm 18$ to $84 \pm 24 \mathrm{~ms}$ after a cumulative doxorubicin dose of $100-120 \mathrm{mg} / \mathrm{m}^{2}$. Greater than $37 \%$ increase in IVRT was $78 \%$ sensitive and $88 \%$ specific for predicting the ultimate development of doxorubicin-induced systolic dysfunction. ${ }^{41}$ In a study of 20 patients receiving a mean cumulative dose of doxorubicin of $211 \pm 82 \mathrm{mg} / \mathrm{m}^{2}$, pulsed tissue Doppler Imaging was reported to show mitral annulus IVRT $<80 \mathrm{~ms}$ in 4 patients who had LVEF $<50 \%$ appeared to outperform both standard Doppler IVRT and basal segment measurements, a finding the authors reported could be of interest to predict later impairment of LV function. ${ }^{42}$ However, the published assessment of the MD Anderson Cancer Center has been that echo diastolic measures are more complex than systolic to obtain and interpret, reproducibility of echocardiographic measures of diastolic function has been problematic, and multiple diastolic measurements have had varying levels of success in identifying early cardiac toxicity. ${ }^{43}$

Echocardiography with dobutamine stress has been used to study contractile reserve in a study of 49 high risk breast cancer patients with poor prognosis. Dobutamine stress echocardiographic detection of reduced contractile reserve, defined as an augmentation of $<5 \mathrm{EF}$ units, was reported to be predictive of a final resting LVEF below 50\% within 18 months. However, the predictive value of reduced contractile reserve for optimizing chemotherapy management and preventing or limiting CHF is unknown. ${ }^{44}$

\section{ECHOCARDIOGRAPHY COMPARED TO CMR}

Limitations of echocardiography compared to CMR for detection of cardiomyopathy have been recently reported by the MD Anderson group in its study of 114 adult survivors of childhood cancer (mean age 39 years) treated with anthracycline chemotherapy and/or chestdirected radiation therapy. ${ }^{45}$ Of the 16 patients (14\%) with LVEF less than $50 \%$ by CMR, Armstrong found 2D echocardiography overestimated mean LVEF of this population by $5 \%$. Compared with CMR, 2D echocardiography (biplane method) had a sensitivity of $25 \%$ and a false-negative rate of $75 \%$ for detection of EF less than $50 \%$, although 3D echocardiography had 53\% and 47\%, respectively. Twelve survivors (11\%) had an EF less than $50 \%$ by CMR but were misclassified as $\geq 50 \%$ (range, $50-68 \%$ ) by 2D echocardiography (biplane method). Detection of cardiomyopathy was improved (sensitivity, 75\%) using a higher 2D echocardiography cutoff $(\mathrm{EF}<60 \%)$ to detect an EF less than $50 \%$ by the reference standard CMR. Despite the issues of accuracy identified by Armstrong, the group continues to regard 2D echocardiography as the screening modality of choice. Armstrong suggests EF values (biplane method) greater than $60 \%$ can be reasonably certain to have normal cardiac function. In this high risk population, survivors with an EF $50-59 \%$ by 2D echocardiography should be considered for comprehensive cardiac assessment, which may include CMR. ${ }^{45}$ 


\section{NOVEL SCINTIGRAPHIC METHODS}

While LVEF measurement by ERNA is regarded widely as the gold standard measurement of cardiotoxicity and can be obtained in virtually all patients with very high reproducibility and low inter-observer variability, decline in LVEF is a relatively late manifestation of myocardial damage and may not preclude late-onset CHF. Techniques that visualize pathophysiologic processes at the tissue level might theoretically enhance detection of cardiotoxicity and potentially augment the ability to predict functional decline and prevent CHF. Novel SPECT techniques for early detection of cardiotoxicity are shown in Table $6 .^{46}$ These novel techniques include static volume indices and functional LV indices by gated SPECT ERNA and MPI, ${ }^{27,28}$ sympathetic neuronal imaging with a wide variety of SPECT and PET tracers, ${ }^{47-55}$ In-111 antimyosin which is a specific marker of myocyte injury and necrosis, ${ }^{54,56,57}$ Tc-99m annexin $\mathrm{V}$ which visualized apoptosis and programmed cell death, ${ }^{58-60}$ fatty acid scintigraphy which visualizes fatty acid retention in the lipid pool of the cytosol which can be impaired by cardiotoxic agents, ${ }^{61}$ and direct imaging of In-111 trastuzumab to study trastuzumab targeting of the myocardium have been considered. ${ }^{62}$ Each of these techniques would require a prospective clinical trial to assess its incremental value in avoiding clinical CHF. ${ }^{46}$ Unfortunately, antimyosin antibody, annexin V, I-123 fatty acids, and In-111 trastuzumab are unavailable for routine clinical use and have limited research availability.

Table 6. Single-photon techniques for early detection of cardiotoxicity

\begin{tabular}{|c|c|}
\hline Technique & Tracer \\
\hline \multirow{2}{*}{$\begin{array}{l}\text { Mechanical pump } \\
\text { function }\end{array}$} & ${ }^{99 \mathrm{~m}}$ Tc ERNA (planar) \\
\hline & ${ }^{99 \mathrm{~m}}$ Tc ERNA (SPECT) \\
\hline Neuronal imaging & ${ }^{123} \mathrm{I} \mathrm{MIBG}$ \\
\hline $\begin{array}{l}\text { Imaging necrosis/ } \\
\text { cell death }\end{array}$ & ${ }^{111}$ In antimyosin \\
\hline $\begin{array}{l}\text { Imaging cell death/ } \\
\text { apoptosis }\end{array}$ & ${ }^{99 \mathrm{~m}}$ Tc annexin $\mathrm{V}$ \\
\hline \multirow[t]{2}{*}{ Fatty acid use } & ${ }^{123}$ I BMIPP \\
\hline & $\begin{array}{l}{ }^{123} \text { I paraphenyl } \\
\text { pentadecanoic acid }\end{array}$ \\
\hline $\begin{array}{l}\text { Therapeutic target } \\
\text { imaging }\end{array}$ & ${ }^{111}$ In trastuzumab \\
\hline
\end{tabular}

Source Ref. 46.

\section{CARDIAC NEURONAL IMAGING}

While serial measurement of resting LVEF is used widely, compensatory myocardial reserve may cause underestimation of myocardial damage and risk of CHF. The progression of anthracycline cardiotoxicity leading to $\mathrm{CHF}$ is associated with a global process of myocardial adrenergic derangement. ${ }^{47}$ Cardiac sympathetic neuronal activity can be imaged noninvasively with I-123 MIBG an analog of norepinephrine among other reported SPECT and PET tracers (Figure 4). Using planar scintigraphy, semi-quantitative analysis of early and late heart to mediastinal $(\mathrm{H} / \mathrm{M})$ ratios can be calculated (Figure 5). Merlet et $\mathrm{al}^{48}$ reported an incremental value of MIBG H/M ratio compared to LVEF for prognosis in CHF. This finding has been sustained by the balance of literature in this field over the past two decades ${ }^{49}$ and both SPECT and PET neurotransmitters ${ }^{46}$ can be hypothesized to augment prediction of risk of chemotherapy-induced CHF. Initial reports have demonstrated MIBG uptake at low dose of doxorubicin prior to and following decline in LV function. ${ }^{50,51}$ A significant decrease in myocardial MIBG uptake at high cumulative doses was observed in nearly all patients with a $10 \%$ reduction of LVEF. ${ }^{51,52}$ These data suggest monitoring drug-induced cardiac sympathetic damage may facilitate recognition of patients at risk of developing CHF who may benefit from early treatment of CHF with or without termination of chemotherapy. ${ }^{51,52}$ Results of the recently published ADMIRE HF trial ${ }^{53}$ and the expectation of impending approval by the FDA of MIBG suggest the potential near-term feasibility of performing these studies.

A major challenge in the design of receptor ligands is to find a ligand that can easily be radiolabeled; has high selectivity and affinity; has high metabolic stability,

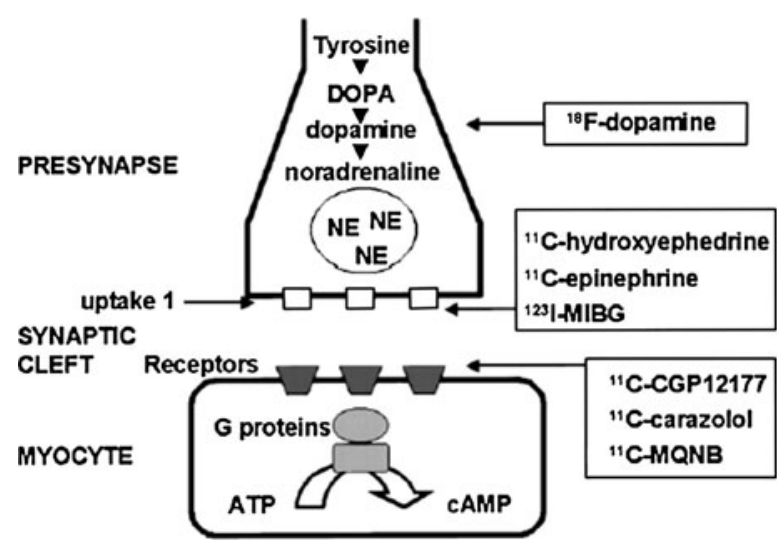

Figure 4. Cardiac sympathetic neuronal metabolic activity provides molecular targets for SPECT and PET imaging tracers. Source Ref. 47. 


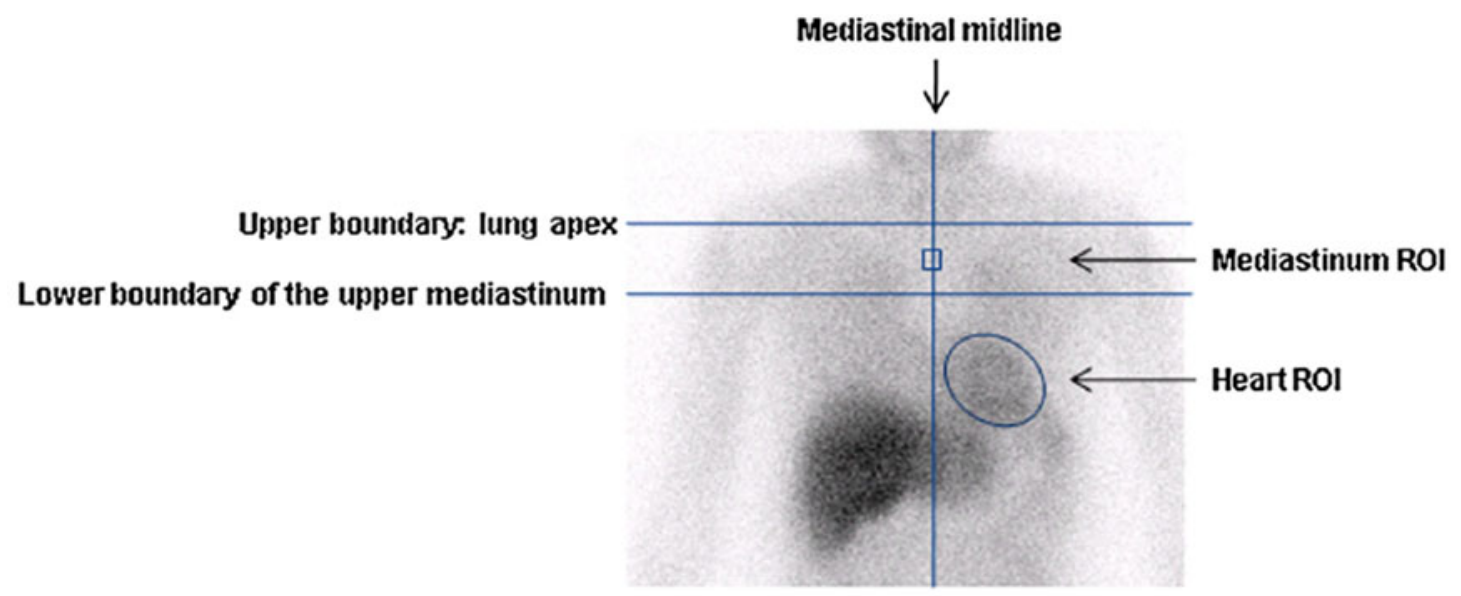

Figure 5. Using planar scintigraphy to quantify heart to mediastinal (H/M) ratios of MIBG uptake indicative of sympathetic nervous system activation in heart failure. Source Ref. 46.

low toxicity, and low lipophilicity, to avoid binding to inactive internalized receptors; and has high specific and low non-specific binding. ${ }^{50}$

\section{INDIUM-11 1 ANTIMYOSIN ANTIBODY}

Indium-111 antimyosin antibody has been used to evaluate doxorubicin cardiotoxicity in adult $t^{54,56}$ and pediatric patients ${ }^{57}$ as well as in myocardial infarction, myocarditis, and cardiac transplant rejection. Antimyosin antibody imaging uptake showed high sensitivity and low specificity and its uptake is apparent in most patients receiving intermediate doses of doxorubicin even in the absence of LV dysfunction. Lack of specificity for prediction of subsequent CHF and lack of ongoing availability of In-111 antimyosin antibody in the United States suggest an unlikely role for it in the foreseeable future.

\section{IMAGING APOPTOSIS WITH ANNEXIN V}

Acute doxorubicin-induced cardiomyopathy based on early apoptosis can be assessed and imaged with annexin V scintigraphy in rats. ${ }^{58}$ This finding makes it possible to use this animal model for repetitive noninvasive evaluation of cardioprotective regimens for anthracycline cardiotoxicity. Apoptosis of myocardial cells plays a critical role in the onset of cardiomyopathy. DOX exposure to endothelial cells and cardiomyocytes caused apoptotic cell death at sub-micromolar concentrations. DOX-induced generation of $\mathrm{H}_{2} \mathrm{O}_{2}$ has been shown to be responsible for this drug's toxicity and apoptosis. $\mathrm{H}_{2} \mathrm{O}_{2}$ in turn enhanced endothelial nitric oxide synthase (eNOS) transcription in endothelial cells and myocytes. Increasing focus on the role of eNOS expression, iron chelation, and iron signaling on DOX-mediated apoptosis has been reviewed, ${ }^{58,59}$ among many other proposed mechanisms of doxorubicininduced cardiotoxicity $\left(\right.$ Table $7^{60}$ ). Increased oxidative stress evidenced by increase in levels of ROS and lipid peroxidation appears to play a prominent role. Reduced expression of cardiac-specific genes, perhaps by affecting expression and function of doxorubicin-sensitive transcriptional regulatory proteins may play a role. Doxorubicin induced apoptosis in vascular cells and cardiomyocytes indicated by caspase activation and inter-nucleosomal DNA degradation. ${ }^{59}$ Specific DNA fragmentation at nucleosomal units is a characteristic biochemical signature of apoptosis. Consideration of apoptotic signal transduction in cardiomyocytes and anti-apoptotic strategies (Figure 6) may facilitate specific molecular markers of the induction, determination, and execution phases of apoptosis. ${ }^{60}$ The specific role of annexin $\mathrm{V}$ imaging in an iron overload experimental rodent model of anthracycline cardiotoxicity has been reported, ${ }^{22}$ and whether annexin $\mathrm{V}$ will play an important clinical role in monitoring anthracycline-induced CHF remains undetermined. ${ }^{60}$ The lack of availability of Annexin V is a substantial barrier for its use in research and clinical application.

\section{FATTY ACIDS}

Taxanes, used to treat breast, lung, and ovarian cancer, can produce ischemia, arrhythmias, and CHF. Taxanes can impair normal microtubular transport systems in cardiomyocytes which results in failure to store free fatty acids (FFA) in the cytosolic lipid pool 


\section{Table 7. Proposed mechanisms of DOX-induced cardiotoxicity}

Inhibition of nucleic acids and protein synthesis

ROS formation and lipid peroxidation

Release of vasoactive amines (histamine, catecholamines, prostaglandins)

Changes in adrenergic function and adenylate cyclase

Inhibition of sarcoplasmic reticulum $\mathrm{Ca}^{2+}$ release

Irreversible reductions in mitochondrial $\mathrm{Ca}^{2+}$ loading and ATP content

Impaired membrane binding, assembly, and activity of mitochondrial creatine kinase

Peroxynitrite-dependent inactivation of mitochondrial creatine kinase or activation of metalloproteinases

Inhibition of membrane-associated calcium-independent phospholipase A2

Reduced expression of

GATA-4

$\alpha$-Actin, myosin light chain 2 slow, myosin heavy chain, tropomyosin, troponin I, troponin C, desmin

$\mathrm{Ca}^{2+}$-ATPase, ryanodine receptor 2

Phospholamban, calsequestrin

Rieske iron-sulfur protein, ADP/ATP translocase, phosphofructokinase, mitochondrial creatine kinase

Phosphorylated form of ERK at the chronic stage

$A D P$, Adenosine diphosphate; ATP, adenosine triphosphate.

Source Ref. 60.

and reducing mitochondrial FFA uptake for beta oxidation. ${ }^{123}$ I-BMIPP and ${ }^{123}$ I-IPPA scintigraphy have been reported to monitor this biochemical perturbation in mitochondrial FFA oxidation without impairing myocardial perfusion. ${ }^{61}$ Taxanes in combination with carboplatin are reported to exert a more profound depression on myocardial FFA metabolism and myocardial contractile dysfunction than doxorubicin alone. The incremental value of reduced ${ }^{123} \mathrm{I}$ BMIPP or ${ }^{123} \mathrm{I}$ IPPA metabolism on prediction of chemotherapyinduced $\mathrm{CHF}$ remains undefined.

\section{IN-111 TRASTUZUMAB}

Trastuzumab cardiotoxicity is potentiated by prior anthracycline exposure. This anthracycline potentiation of trastuzumab cardiotoxicity is believed to result from an initial increase in myocardial HER2 expression followed by inhibition of HER2-mediated signaling resulting in ATP depletion, contractile dysfunction, and also immune-mediated destruction. ${ }^{46}$ Early uptake of In111 trastuzumab in anthracycline-exposed patients has been suggested in one preliminary study to identify in a pre-symptomatic stage the presence of early cardiotoxicity. ${ }^{62}$ The value of this approach for monitoring patients at risk for trastuzumab induced CHF in anthracycline-pretreated patients remains undefined.

\section{BIOMARKERS}

Troponin elevation following anthracycline chemotherapy is predictive of larger and more sustained decline in LVEF compared to those without troponin elevation. ${ }^{63}$ Cardinale and Sandri ${ }^{63}$ has proposed the potential role of troponin as a standard marker in identifying patients at risk of cardiotoxicity, and has advocated use of angiotensin-converting enzyme inhibitors, angiotensin II type 1a receptor blockers, and/or carvedilol $^{64}$ in preventing cardiac dysfunction and cardiac events in at risk patients. However, at this time no clarity exists as to recommended timing and guidelines for use of biomarkers and their role in modifying chemotherapy. Recently, elevations in both cardiac biomarkers NT-pro-BNP and high sensitive cardiac TnT were found before echocardiographic evidence of systolic and diastolic dysfunction. ${ }^{65}$ The authors suggest persistent elevations in NT-pro-BNP and hs-cTnT concentrations simultaneously for a period exceeding 14 days might be used for identification of patients at risk of developing cardiotoxicity and requiring further cardiological follow-up.

Other studies have not shown consistent correlation between troponin elevation and administration of anthracycline or Herceptin. ${ }^{66-68}$ While an increase in pro-BNP has been reported early after anthracycline administration, elevated pro-BNP has been found not to be predictive of future LV dysfunction. Pharmacogenomics has recently emerged as a potential biomarker of chemotherapy-induced cardiac dysfunction. ${ }^{69}$ Study of 2,977 single-nucleotide polymorphisms (SNPs) in 220 key drug biotransformation genes was studied in 156 children. Multiple genetic variants in SLC28A3 and other genes were found to be associated with anthracycline-induced cardiotoxicity. Combined with clinical risk factors, this study suggests that genetic risk profiling might be used to identify high risk patients who can then be provided with 


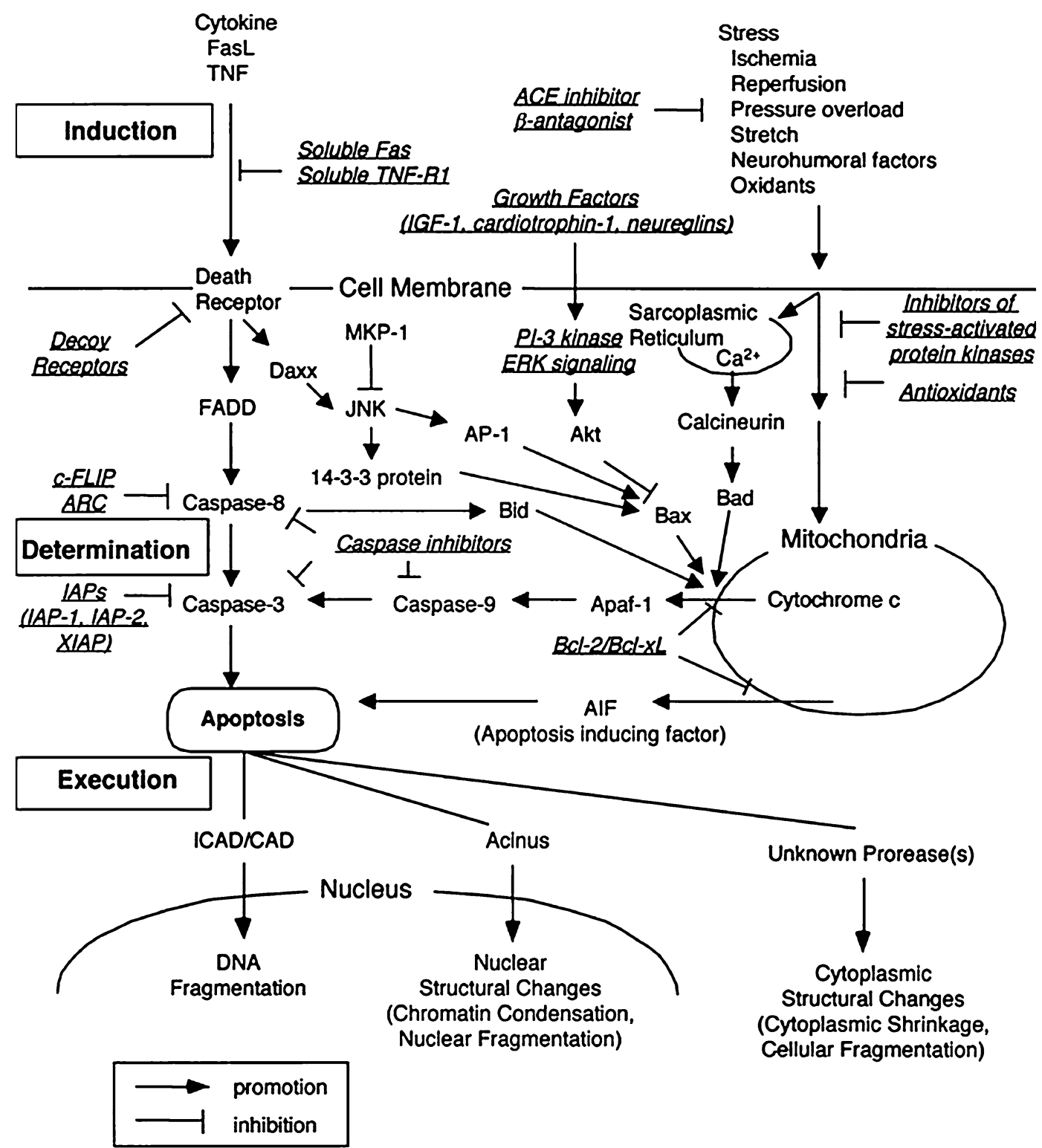

Figure 6. Apoptotic signal transduction in cardiomyocytes and anti-apoptotic strategies. Source Ref. 60.

safer treatment options. While they are inexpensive and readily available, no consensus on the role of biomarkers for prediction of anthracycline cardiotoxicity in adults has emerged. ${ }^{66-69}$ The role of biomarkers is being currently evaluated in the multicenter $\mathrm{NIH}$ funded PREDICT study. ${ }^{70}$ Biomarkers in 2013 remain of great promise and investigative interest.

\section{LONGITUDINAL STRAIN}

Echocardiographic strain (S) and strain rate imaging (SRI) analyses, alone or in combination with biomarkers, have been reported to identify pre-symptomatic anthracycline cardiotoxicity in small series of patients. ${ }^{71-74}$ Jurcut et $\mathrm{al}^{71}$ demonstrated Doppler-based SRI, but not myocardial velocity imaging or conventional echocardiography, is a sensitive tool capable of documenting small, clinically inapparent but significant changes in cardiac function in a pilot study of 16 elderly patients with histologically proven early breast cancer with normal LVEF who received six courses of pegylated liposomal doxorubicin (Figure 7). The investigators suggested Doppler-based myocardial deformation imaging should be used for cardiac function monitoring 

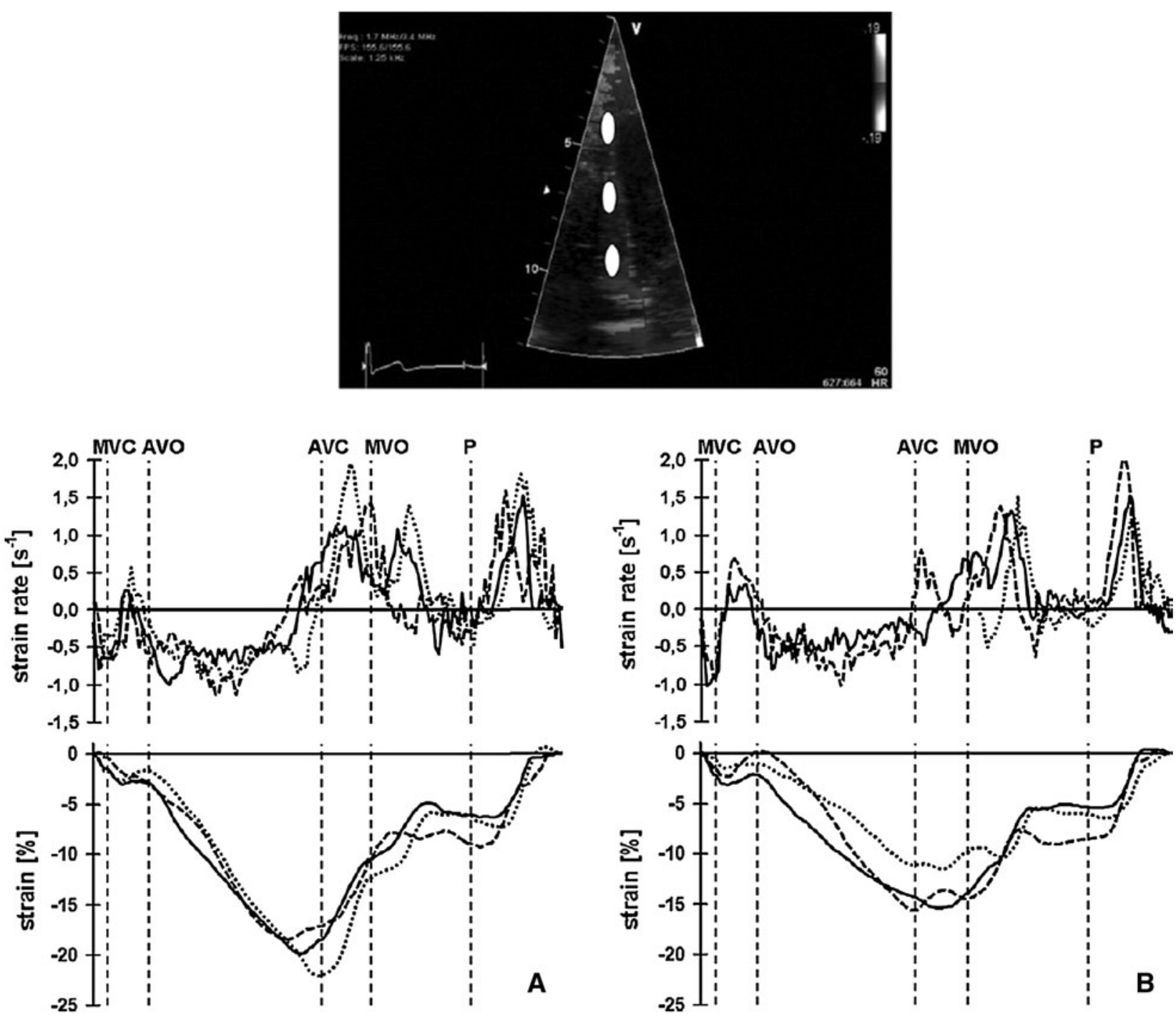

Figure 7. Echo strain rate (SR) imaging identifies decreased peak systolic longitudinal SR (top) and $\mathrm{S}$ (bottom) in the basal (continuous line), mid (interrupted line) and apical (dotted line) segments of the inferoseptal wall in a patient after six cycles (B) vs baseline (A). White circles in the image show basal, mid, and apical regions of interest where deformation data were derived. $A V C$, Aortic valve closure; $A V O$, aortic valve opening; $M V C$, mitral valve closure; $M V O$, mitral valve opening; $P$, beginning of $\mathrm{P}$ wave. Source Ref. 71.

during chemotherapy, although this pilot study did not address issues of CHF prediction directly. Subtle abnormalities of systolic and diastolic function were present in patients with asymptomatic breast cancer with prior anthracycline exposure. The specificity of longitudinal strain assessment in patients receiving chemotherapy may be reduced by obesity, valvular heart disease, infiltrative disease, LV hypertrophy, myocardial infarction, age and gender as recently reviewed. ${ }^{75}$

Cardiac troponin plasma concentrations and longitudinal strain predicted the development of cardiotoxicity in patients treated with anthracyclines and trastuzumab, and these two parameter(s) have been suggested to detect chemotherapy-treated patients who may benefit from alternative therapies. ${ }^{73}$ A recent study of 81 women with breast cancer treated with anthracyclines, taxanes, and trastuzumab concluded the 5 patients who developed CHF during the 15-month follow-up period were successfully identified by peak systolic strain and ultrasensitive troponin I measured at the completion of treatment despite a decline of echocardiographic LVEF (64-59\%) within normal limits. ${ }^{74}$ Peak systolic longitudinal myocardial strain decreased from -21 to -19 but in the women who developed chemotherapy-induced cardiotoxicity, the 
mean longitudinal strain decreased to -15 . Thus, longitudinal strain below $-19 \%$ was present in all those patients who later developed CHF and longitudinal strain appeared to predict decreases of LVEF below $50 \%$. In contrast, no predictive value of radial strain was found, possibly due to the variability of the measurement. ${ }^{74}$ Biomarkers NT-proBNP and ST2 did not predict cardiotoxicity ${ }^{74}$ Predictive accuracy of longitudinal strain and the biomarker TnI has been critically reviewed by Lipshultz who observed the incremental value of the strain rate compared to other predictor variables by ROC analysis was undefined. ${ }^{76}$ These early results suggest the need for larger prospective studies compared to quantitative LVEF and volume indices by ERNA to detect chemotherapy-induced cardiotoxicity and risk of CHF as well as monitoring the value of cardiac therapy with ACE inhibitors and the beta blocker carvedilol for asymptomatic LV dysfunction. Long-term, large-scale outcome studies with hard clinical end points will be required to determine the clinical significance of these findings and the ability of these measurements to improve patient outcomes by directing changes in therapy to optimize antineoplastic efficacy while preventing CHF, thereby leading to improvements in quality and quantify of life.

\section{CONSIDERATIONS IN CHILDREN}

Children appear to be more susceptible than adults to the cardiotoxic effects of anthracycline therapy, although there is considerable variation in the individual susceptibility to these side effects. ${ }^{2,10-14,57,69,77-80}$ Children with Hodgkin's disease have been reported to manifest cardiotoxicity early and at low cumulative doses of doxorubicin. ${ }^{77}$ The potentially long latency and high cumulative incidence of chronic cardiac dysfunction associated with cancer treatment indicates the need for long-term monitoring of asymptomatic children. ERNA was reported to be more sensitive than echocardiography in detecting early impairment of LV function and was recommended for baseline and serial assessment of LV function in children with Hodgkins disease treated with doxorubicin. ${ }^{77}$ This recent report supports the observation of the complementary nature of ERNA and echocardiography and the recommendations of the Children's Cancer Study Group for monitoring with both techniques (Figure 3). ${ }^{10}$

Kremer et $\mathrm{al}^{57}$ found evidence of myocardial injury as measured by cardiac uptake of ${ }^{111} \mathrm{In}$-antimyosin in children with normal fractional shortening by echocardiography. These results are consistent with endomyocardial biopsy findings and serum cardiac troponin concentrations measured in patients receiving anthracycline therapy. As in adults, while more sensitive for the detection of subclinical cardiotoxicity, much like the endomyocardial biopsy, a role of these markers including ${ }^{111}$ In-antimyosin imaging remains undefined. As with adults, lack of current availability of ${ }^{111} \mathrm{In}$ antimyosin in the United States has limited its potential role.

Late cardiotoxicity in children and young adults may be related to acute cardiac damage during treatment. Another conceivable mechanism is damage by radiation and chemotherapy to cardiac and bone marrow-derived stem cells and endothelial progenitor cells and the impairment of downstream repopulation of cardiovascular targets. Monitoring of cell signaling and migration of bone marrow-derived stem cells may provide insights into the mechanism of cardiotoxicity following chemotherapy in childhood and offer opportunities to identify therapeutic response to stem cell therapies for chemotherapy-induced cardiotoxicity. Using PET direct cell labeling or reporter gene-based cell labeling may be considered to develop new methods of tracking stem cell regenerative cardiac therapy for chemotherapy-induced cardiotoxicity in children and adults. ${ }^{81}$

\section{CARDIAC MAGNETIC RESONANCE IMAGING}

CMR is now recognized by the ACC/AHA as a method to screen for chemotherapy-related cardiotoxicity. ${ }^{82}$ Key advantages include accuracy and reproducibility of LV and RV volumes and EF, and the ability to visualize preclinical myocardial changes prior to the onset of LV dysfunction, increased $\mathrm{T} 2$ weighted images associated with tissue edema resulting from acute myocardial inflammation and injury as seen in myocarditis. ${ }^{83} \mathrm{~A}$ characteristic pattern of mid myocardial hyperenhancement has been reported in breast cancer patients receiving trastuzumab who experienced $\mathrm{LV}$ dysfunction. ${ }^{84} \mathrm{~T} 1$ weighted hyperenhancement within 3 days of the first anthracycline administration was associated with decreased LVEF on day 28 after starting chemotherapy. ${ }^{85}$ Clinical trials of T1 weighted CMR imaging to assess cardiotoxicity and predict LV dysfunction and CHF appear warranted.

An experimental rodent myocardial study of early detection of doxorubicin cardiotoxicity has correlated signal intensity of gadolinium enhancement with the dose-related degree of myocardial vacuolization and decline in LVEF. ${ }^{86}$ A new superparamagnetic iron oxide probe conjugated to recombinant human annexin has demonstrated diffuse myocardial signal loss in rats treated with doxorubicin, suggestive of apoptosis by $\mathrm{CMR}^{87,88}$ Myocardial fibrosis detected as delayed enhancement (DE) by CMR has proven prognostic value in coronary heart disease, ${ }^{89}$ and restrictive myocardial diseases including aortic stenosis, ${ }^{90}$ hypertrophic cardiomyopathy, ${ }^{91,92}$ and the infiltrative diseases of sarcoidosis ${ }^{93}$ and amyloidosis. ${ }^{94}$ CMR studies in larger populations of patients receiving potentially cardiotoxic agents are needed to see whether the presence of fibrosis 
detected by gadolinium enhancement identifies a cohort of patients more vulnerable to the cardiotoxic effects of chemotherapy, who may benefit from more frequent and long-term follow-up, and earlier treatment with ACE inhibitors and beta blockade to reduce the incidence and severity of chemotherapy-induced heart failure.

Thus, CMR detection of anthracycline cardiotoxicity appears to hold promise for further clinical investigation.

\section{Molecular Targets}

An intriguing variety of molecular targets offer potential new insights for detection and management of chemotherapy-induced cardiotoxicity. While a detailed review of these molecular mechanisms is beyond the scope of this review, it seems clear a better understanding of the molecular mechanisms of anthracyclineinduced tumor cell and cardiomyocyte dysfunction and/ or death may permit the development of strategies to widen the therapeutic index of this class of agents. ${ }^{95}$ The development of new therapeutic strategies based on NRG-1, such as the delivery of nucleotides that inhibit miR-146a, is promising for treating heart failure in patients exposed to anthracyclines. ${ }^{96}$ Delineating control mechanisms such as caspase-dependent down-regulation of doxorubicin-induced myocardial apoptosis accompanying postnatal heart maturation ${ }^{97}$ may offer fine tuning of dose intensity and dosing interval to optimize antineoplastic and cardioprotective goals of therapy. Exploring the pathways by which doxorubicin inhibits transcription of the SERCA2 gene and affects abnormal calcium handling and cardiac dysfunction observed in doxorubicin cardiomyopathy is yet another avenue for exploration of the underlying molecular aberrations potentially causing cardiac dysfunction. ${ }^{98}$

\section{CONCLUSIONS AND FUTURE DIRECTIONS}

Serial monitoring of radionuclide LVEF continues to provide the evidence-based standard for management of risk of $\mathrm{CHF}$ associated with anthracycline therapy. An important priority is to develop treatment and monitoring strategies to eliminate late-onset LV dysfunction and $\mathrm{CHF}$, of particular concern to children and to all long-term cancer survivors whose lifetime risk of CHF may be substantial. The role of gated SPECT ERNA appears very promising for its high degree of accuracy and reproducibility, tracking of RV and LV function, and LV volume indices. The current generation of high-speed solid-state digital gamma cameras provides an opportunity to perform SPECT ERNA studies using a significantly lower dose of radiotracer, which is particularly appealing in pediatric patient population. The unmeasurably very low theoretical cancer risk of exposure to low dose radiotracers of nuclear cardiology studies must be evaluated clinically relative to the substantial proven clinical benefit of this monitoring approach to detecting cardiotoxicity and preventing $\mathrm{CHF}^{99}$ Echocardiography is widely available but has limitations of poor acoustic windows and geometric assumptions in the calculation of LVEF and its safety and effectiveness for prevention of $\mathrm{CHF}$ in high risk populations receiving chemotherapy remains inadequately evaluated. A need exists for rigorous, blinded analysis of the ability of echocardiography to assess serial changes in LVEF and prevent CHF in patients receiving chemotherapy and to identify a set of guidelines with echocardiography by which chemotherapy can be managed, as demonstrated by the radionuclide monitoring experience. The value of $3 \mathrm{D}$ echo and contrast echo can be reasonably expected to improve the accuracy of blinded echocardiographic EF measurements by avoiding underestimation of LV volumes by 2D echocardiography, as reviewed by the American Society of Echocardiography. In the best interests of optimizing patient outcome, results of technically limited studies by any technique should be verified by a complementary modality.

The role of volume indices, phase analysis, diastolic function, and isovolumetric indices of cardiac function, cardiac sympathetic neuronal imaging with ${ }^{123}$ I-MIBG, biomarkers, annexin $\mathrm{V}$ imaging of apoptosis, and echo strain imaging remain potential methods to expand our repertoire of diagnostic tools to develop management guidelines to prevent CHF. The current lack of tracer availability of MIBG, annexin V, and antimyosin antibody is problematic. Given the special needs of children and adults who survive their cancers and live to face potential life long morbidity of $\mathrm{CHF}$, correlation of functional and molecular imaging targets with long-term $\mathrm{CHF}$ risk remain an important obligation of this field of cardio-oncology. Clinical assessment of the contributions of different chemotherapeutic agents with different pathophysiologic models of cardiotoxic risk require careful clinical judgment as well as traditional and novel methods to assess benefits and risk of modern chemotherapy. Recent reports of chemotherapy-induced hypertension as a common adverse effect of angiogenesis inhibitors and immunosuppressants demonstrate the need for routine BP monitoring and guideline-based management of hypertension. Developing and validating with blinded outcome studies new techniques to manage chemotherapy by monitoring cardiotoxicity at the tissue and cellular levels to prevent $\mathrm{CHF}$ holds promise for enhancing quality and quantity of life for cancer survivors.

The current era offers great promise for the development of safer and more potent chemotherapeutic agents and numerous methods to optimize detection of 
the critical mass of cardiotoxicity and LV dysfunction that predicts risk of $\mathrm{CHF}$ with continued therapy. Enhanced complexity of cardio-oncology is evident given the broader range of chemotherapies and mechanisms of cardiotoxicity, the higher dosages, the extremes of age, the enhanced survival of the patients we routinely treat. These factors expose our patients to longer periods of vulnerability to chemotherapy-induced cardiotoxicity and CHF. In our quest to optimize detection of cardiotoxicity, the challenge for the field of cardio-oncology is to move beyond phenomenology of cardiotoxicity to provide and validate outcome-based guidelines that effectively mitigate risk of $\mathrm{CHF}$ while optimizing chemotherapeutic benefit. Cancer genomics appears promising not only for staging of cancer but also for identifying specific markers of cardiotoxicity, as with kinase inhibitors. ${ }^{100}$

As we wait for development and validation of the clinical effectiveness of traditional and novel candidate methods, serial monitoring of LVEF by ERNA and chemotherapy management concordant with published guidelines remains a reliable and cost-effective means of limiting CHF and reducing the incidence and severity of chemotherapy-induced cardiomyopathy in adults and children. ${ }^{6-11,101}$ As we enter the "uncharted waters" of greater use of kinase inhibitors which promise to further complicate the landscape of potential cardiotoxicity, ${ }^{100} \mathrm{a}$ multimodality surveillance approach appears to make sense to enhance safety and effectiveness of chemotherapy. Below and in Table 8, we suggest implementation of a novel multimodality integrative imaging approach which aims to protect patients using the strength of evidencebased methodologies in parallel with other techniques that promise to detect risk of subsequent HF more sensitively than traditional LVEF measurements allow.

\section{RECOMMENDATION: MULTIMODALITY INTEGRATIVE APPROACH TO MONITORING CHEMOTHERAPY AND RADIATION-INDUCED LV DYSFUNCTION}

A new multi-modality monitoring approach is proposed which integrates evidence-based strengths of CMR, echocardiography, ERNA and BP management

Table 8. Recommendation: multimodality integrative approach to monitoring chemotherapy and radiation-induced LV dysfunction

1. Serial surveillance monitoring of LVEF precedes initiation and each cycle of chemotherapy and alternates between echocardiography and ERNA techniques

2. Recommended thresholds of LVEF are $\leq 60 \%$ by $2 \mathrm{D}$ echocardiography, $\leq 55 \%$ by SPECT ERNA or contrast $2 \mathrm{D}$ or $3 \mathrm{D}$ echocardiography, and $\leq 50 \%$ by planar ERNA

3. Tissue Doppler imaging, longitudinal strain imaging more positive than $-18 \%$, and enlarging left atrial volumes $>25 \%$ above the upper limit of normal by echo or CMR, reflecting progressive diastolic dysfunction; Troponin $\mathrm{T}$ and NT-proBNP can be prospectively monitored to assess the potential value of these markers for detecting subclinical progressive restrictive cardiomyopathy and myocytolysis that may predict clinical $\mathrm{HF}$

4. High-speed CZT SPECT-gated ERNA (GBPS) imaging with ultra low dose radiotracer $(<10 \mathrm{mCi}$ Tc-99m pertechnetate, $<10 \mathrm{mSv}$ exposure, $<10$-minute imaging time) can be employed in the subset of patients who have access to this advanced and increasingly available technology

5. Performance of tissue characterization to detect hyperenhancement evidence of fibrosis or infiltrative restrictive myocardial disease is recommended for any patient whose baseline or follow-up LVEF by ERNA or echocardiography declines to threshold, and any patient with prolonged QTC $>0.5$ seconds on resting ECG

6. Monitor ESVI by all techniques. Patients with ESVI $>35 \mathrm{~mL} / \mathrm{m}^{2}$ by any technique should be treated with ACE inhibitor/ARB and carvedilol

7. Patients whose LVEF declines by $\geq 10 \mathrm{EF}$ units below the threshold EF would be treated with carvedilol and ACE inhibitor/ARB and undergo (repeat) CMR

8. Patients with GFR $<30$ who are not candidates for gadolinium CMR studies and any patient who develops CHF would be treated with carvedilol and ACE inhibitor/ARB

9. Consider endomyocardial biopsy to resolve questions regarding the etiology of heart failure or decline of LVEF $>10 \mathrm{EF}$ units below $\mathrm{EF}$ threshold in unusual cases when co-existing pathology such as an infiltrative disease (e.g., sarcoid) confounds the differential diagnosis

10. Therapy: (A) Close serial monitoring of BP and rigorous, guideline-based treatment of hypertension to current JNC recommended targets initiating therapy with Carvedilol and ACE-I/ARB. (B) Discontinue chemotherapy with decline of LVEF below $10 \mathrm{EF}$ units to threshold LVEF or below, increase of LVESVI $>45 \mathrm{~mL} / \mathrm{m}^{2}$, increase in left atrial volume $>25 \%$ upper normal for gender and BSA 
and biomarkers for surveillance and validation of cardiotoxicity and prevention of clinical heart failure in patients receiving a broad spectrum of cancer therapies (Table 8). The strategy exploits the wide availability and complementary strengths of echocardiography and ERNA for surveillance of cardiotoxicity reinforced by CMR for validation and identification of restrictive myocardial disease while ensuring close monitoring and treatment of BP and early preclinical signs of cardiotoxicity to prevent $\mathrm{CHF}$.

1. Serial surveillance monitoring of LVEF precedes initiation and each cycle of chemotherapy and alternates between echocardiography and ERNA techniques.

2. Recommended thresholds of LVEF are $\leq 60 \%$ by $2 \mathrm{D}$ echocardiography, $\leq 55 \%$ by SPECT ERNA or contrast $2 \mathrm{D}$ or $3 \mathrm{D}$ echocardiography, and $\leq 50 \%$ by planar ERNA.

3. Tissue Doppler imaging, longitudinal strain imaging more positive than $-18 \%$, and enlarging left atrial volumes $>25 \%$ above the upper limit of normal by echo or CMR, reflecting progressive diastolic dysfunction; Troponin T and NT-proBNP can be prospectively monitored to assess the potential value of these markers for detecting subclinical progressive restrictive cardiomyopathy and myocytolysis that may predict clinical HF.

4. High-speed CZT SPECT-gated ERNA (GBPS) imaging with ultra low dose radiotracer $(<10 \mathrm{mCi}$ Tc-99m pertechnetate, $<10 \mathrm{mSv}$ exposure, $<10$ minute imaging time) can be employed in the subset of patients who have access to this advanced and increasingly available technology.

5. Performance of tissue characterization to detect hyperenhancement evidence of fibrosis or infiltrative restrictive myocardial disease is recommended for any patient whose baseline or follow-up LVEF by ERNA or echocardiography declines to threshold, and any patient with prolonged QTc $>0.5$ seconds on resting ECG.

6. Monitor ESVI by all techniques. Patients with ESVI $>35 \mathrm{~mL} / \mathrm{m}^{2}$ by any technique should be treated with ACE inhibitor/ARB and carvedilol.

7. Patients whose LVEF declines by $\geq 10$ EF units below the threshold EF would be treated with carvedilol and ACE inhibitor/ARB and undergo (repeat) CMR.

8. Patients with GFR $<30$ who are not candidates for gadolinium CMR studies and any patient who develops CHF would be treated with carvedilol and ACE inhibitor/ARB.

9. Consider endomyocardial biopsy to resolve questions regarding the etiology of heart failure or decline of LVEF >10 EF units below EF threshold in unusual cases when co-existing pathology such as an infiltrative disease (e.g., sarcoid) confounds the differential diagnosis.

10. Therapy: (A) Close serial monitoring of BP and rigorous, guideline-based treatment of hypertension to current JNC recommended targets initiating therapy with Carvedilol and ACE-I/ARB. (B) Discontinue chemotherapy with decline of LVEF below $10 \mathrm{EF}$ units to threshold LVEF or below, increase of LVESVI $>45 \mathrm{~mL} / \mathrm{m}^{2}$, increase in left atrial volume $>25 \%$ upper normal for gender and BSA.

\section{References}

1. Yeh ETH, Bickford CL. Cardiovascular complications of cancer therapy: Incidence, pathogenesis, diagnosis, and management. J Am Coll Cardiol 2009;53:2231-47.

2. Carver JR, Shapiro CL, Ng A, Jacobs L, Schwartz C, Virgo KS, et al. American Society of Clinical Oncology clinical evidence review on the ongoing care of adult cancer survivors: cardiac and pulmonary late effects. J Clin Oncol 2007;25:3991-4008.

3. Mouhayar E, Salahudeen A. Hypertension in cancer patients. Tex Heart Inst J 2011;38:263-5.

4. Maitland ML, Bakris GL, Black HR, Chen HX, Durand J-B, Elliott WJ, et al. Cardiovascular Toxicities Panel, convened by the Angiogenesis Task Force of the National Cancer Institute Investigational Drug Steering Committee. Initial assessment, surveillance, and management of blood pressure in patients receiving vascular endothelial growth factor signaling pathway inhibitors. J Natl Cancer Inst 2010;102:596-604.

5. Lefrak EA, Pit'ha J, Rosenheim S, Gottlieb JA. A clinicopathologic analysis of Adriamycin cardiotoxicity. Cancer 1973;32: 302-14.

6. Alexander J, Dainiak N, Berger H, Goldman L, Johnstone D, Reduto L, et al. Serial assessment of doxorubicin cardiotoxicity with quantitative radionuclide angiocardiography. N Engl J Med 1979;300:278-83.

7. Choi BW, Hi B, Schwartz PE, Alexander I, Wackers FJTh, Gottschalk A, et al. Serial radionuclide assessment of doxorubicin cardiotoxicity in cancer patients with abnormal baseline resting left ventricular performance. Am Heart J 1983;106:638-43.

8. Schwartz RG, McKenzie WB, Alexander J, Sager P, D'Souza A, Manatunga $\mathrm{A}$, et al. Congestive heart failure and left ventricular dysfunction complicating doxorubicin therapy: A seven year experience using serial radionuclide angiocardiography. Am J Med 1987;82:1109-18.

9. Mitani I, Jain D, Joska TM, Burtness B, Zaret BL. Doxorubicin cardiotoxicity: Prevention of congestive heart failure with serial cardiac function monitoring with equilibrium radionuclide angiocardiography in the current era. J Nucl Cardiol 2003;10: 132-9.

10. Steinherz LJ, Graham T, Hurwitz R, Sondheimer HM, Schwartz RG, Shaffer EM, et al. Guidelines for cardiac monitoring of children during and after anthracycline therapy: Report of the Cardiology Committee of the Childrens Cancer Study Group. Pediatrics 1992;89:942-9.

11. Steinherz LJ, Steinherz PG, Tan CT, et al. Cardiac toxicity 4 to 20 years after completing anthracycline therapy. JAMA 1991; $266: 1672-7$ 
12. Lipshultz SE, Colan SD, Gelber RD, et al. Late cardiac effects of doxorubicin therapy for acute lymphoblastic leukemia in childhood. N Engl J Med 1991;324:808-15.

13. Lipshultz SE, Lipsitz SR, Sallan SE, et al. Chronic progressive cardiac dysfunction years after doxorubicin therapy for childhood acute lymphoblastic leukemia. J Clin Oncol 2005;23:2629-36.

14. Cameron EH, Lipshultz SE, Tarbell NJ, et al. Cardiovascular disease in long term survivors of pediatric Hodgkin's disease. Prog Pediatr Cardiol 1998;8:139-44.

15. Plana JC. Chemotherapy and the heart. Rev Esp Cardiol 2011; 64:409-15

16. Billingham ME, Mason JW, Bristow MR, Daniels JR. Anthracycline cardiomyopathy monitored by morphologic changes. Cancer Treat Rep 1978;62:865-72.

17. Von Hoff DD, Layard M. Risk factors for development of daunorubicin cardiotoxicity. Cancer Treat Rep 1981;65(Suppl 4):19-23.

18. Ewer MS, Vooletich MT, Durand JB, Woods ML, Davis JR, Valero $\mathrm{V}$, et al. Reversibility of trastuzumab-related cardiotoxicity: New insights based on clinical course and response to medical treatment. J Clin Oncol 2005;23:7820-6.

19. Panjrath GS, Jain D. Trastuzumab-induced cardiac dysfunction. Nucl Med Commun 2007;28:69-73.

20. Bristow MR, Lopez MB, Mason JW, Billingham ME, Winchester MA. Efficacy and cost of cardiac monitoring in patients receiving doxorubicin. Cancer 1982;50:32-41.

21. Lindenfeld J, et al. Executive summary: HFSA 2010 comprehensive heart failure practice guideline. J Cardiac Fail 2010;16: 2010.

22. Panjrath GS, Patel V, Valdiviezo CI, Narula N, Narula J, Jain D. Potentiation of doxorubicin cardiotoxicity by iron loading in a rodent model. J Am Coll Cardiol 2007;49:2457-64.

23. Mackey JR, Clemons M, Cote MA, et al. Cardiac management during adjuvant trastuzumab therapy: Recommendations of the Canadian Trastuzumab Working Group. Curr Oncol 2008;15:24-35.

24. Anand AJ. Fluorouracil cardiotoxicity. Ann Pharmacother 1994;28:374

25. Akhtar SS, Salim KP, Bano ZA. Symptomatic cardiotoxicity with high-dose 5-fluorouracil infusion: A prospective study. Oncology 1993;50:441.

26. Palmeri ST, Bonow RO, Myers CE, Seipp C, Jenkins J, Green $\mathrm{MV}$, et al. Prospective evaluation of doxorubicin cardiotoxicity by rest and exercise radionuclide angiography. Am J Cardiol 1986;58:607-13.

27. Van Kriekinge SD, Berman DS, Germano G. Automatic quantification of left ventricular ejection fraction from gated blood pool SPECT. J Nucl Cardiol 1999;6:498-506.

28. Wexler O, Yoder SR, Elder JL, Mackin ML, Chen L, Mixon L, et al. Effect of gender on cardiovascular risk stratification with ECG gated SPECT left ventricular volume indices and ejection fraction. J Nucl Cardiol 2009;16:28-37.

29. White HD, Norris RM, Brown MA, Brandt PW, Whitlock RM, Wild CJ. Left ventricular end-systolic volume as the major determinant of survival after recovery from myocardial infarction. Circulation 1987;76:44-51.

30. Daou D, Harel F, Helal BO, et al. Electrocardiographically gated blood-pool SPECT and left ventricular function: Comparative value of 3 methods for ejection fraction and volume estimation. J Nucl Med 2001;42:1043-9.

31. Hambye AS, Vervaet A, Dobbeleir A. Variability of left ventricular ejection fraction and volumes with quantitative gated SPECT: Influence of algorithm, pixel size and reconstruction parameters in small and normal-sized hearts. Eur J Nucl Med Mol Imaging 2004;31:1606-13.
32. Hesse B, Lindhardt TB, Acampa W, Anagnostopoulos C, Ballinger J, Bax JJ, et al. EANM/ESC guidelines for radionuclide imaging of cardiac function. Eur $\mathrm{J}$ Nucl Med Mol Imaging 2008;35:851-85.

33. Hendel RC, Berman DS, Di Carli MF, Heidenreich PA, Henkin RE, Pellikka PA, et al. ACCF/ASNC/ACR/AHA/ASE/SCCT/ SCMR/SNM 2009 appropriate use criteria for cardiac radionuclide imaging: A Report of the American College of Cardiology Foundation Appropriate Use Criteria Task Force, the American Society of Nuclear Cardiology, the American College of Radiology, the American Heart Association, the American Society of Echocardiography, the Society of Cardiovascular Computed Tomography, the Society for Cardiovascular Magnetic Resonance, and the Society of Nuclear Medicine Endorsed by the American College of Emergency Physicians. J Am Coll Cardiol 2009;53:2201-29.

34. Cottin Y, Touzery C, Dalloz F, et al. Comparison of epirubicin and doxorubicin cardiotoxicity induced by low doses: Evolution of the diastolic and systolic parameters studied by radionuclide angiography. Clin Cardiol 1998;21:665-70.

35. Groch MW, DePuey EG, Belzberg AC, Erwin WD, Kamran M, Barnett CA, et al. Planar imaging versus gated blood-pool SPECT for the assessment of ventricular performance: A multicenter study. J Nucl Med 2001;42:1773-9.

36. McManus DD, Shah SJ, Fabi MR, Rosen A, Whooley MA, Schiller NB. Prognostic value of left ventricular end-systolic volume index as a predictor of heart failure hospitalization in stable coronary artery disease: Data from the heart and soul study. J Am Soc Echocardiogr 2009;22:190-7. doi:10.1016/ j.echo.2008.11.005.

37. Mulvagh SL, Rakowski H, Vannan MA, Abdelmoneim SS, Becher H, Bierig SM, et al. American Society of Echocardiography consensus statement on the clinical applications of ultrasonic contrast agents in echocardiography. J Am Soc Echocardiogr 2008;21:1179-201.

38. Thavendiranathan P, Grant AD, Negishi T, Plana JC, Popovic' ZB, Marwick TH. Reproducibility of echocardiographic techniques for sequential assessment of left ventricular ejection fraction and volumes: Application to patients undergoing cancer chemotherapy. J Am Coll Cardiol 2013;61:77-84.

39. Mor-Avi V, Lang RM. Is echocardiography reliable for monitoring the adverse cardiac effects of chemotherapy? J Am Coll Cardiol 2013;61:85-7.

40. Marchandise B, et al. Early detection of doxorubicin cardiotoxicity: Interest of Doppler echocardiographic analysis of left ventricular filling dynamics. Am Heart J 1989;118:92-8.

41. Stoddard MF, Seeger J, Liddell NE, Hadley TJ, Sullivan DM, Kupersmith J. Prolongation of isovolumetric relaxation time as assessed by Doppler echocardiography predicts doxorubicininduced systolic dysfunction in humans. J Am Coll Cardiol 1992;20:62-9.

42. Tassan-Mangina S, Codorean D, Metivier M, Costa B, Himberlin C, Jouannaud C, et al. Tissue Doppler imaging and conventional echocardiography after anthracycline treatment in adults: Early and late alterations of left ventricular function during a prospective study. Eur J Echocardiogr 2006;7:141-6.

43. Banchs J, Jefferies JL, Plana JC, Hundley WG. Imaging for cardiotoxicity in cancer patients. Tex Heart Inst J 2011;38:268-9.

44. Civelli M, Cardinale D, Martinoni A, Lamantia G, Colombo N, Colombo A, et al. Early reduction in left ventricular contractile reserve detected by dobutamine stress echo predicts high-dose chemotherapy-induced cardiac toxicity. Int J Cardiol 2006; 111:120-6. 
45. Armstrong GT, Plana JC, Zhang N, Srivastava D, Green DM, Nes KK, et al. Screening adult survivors of childhood cancer for cardiomyopathy: Comparison of echocardiography and cardiac magnetic resonance imaging. J Clin Oncol 2012. doi:10.1200/ JCO.2011.40.3584.

46. de Geus-Oei LF, Mavinkurve-Groothuis AMC, Bellersen L, Gotthardt M, Oyen WJG, Kapusta L, et al. Scintigraphic techniques for early detection of cancer treatment-induced cardiotoxicity. J Nucl Med 2011;52:560-71.

47. Carrio I. Cardiac neurotransmission imaging. J Nucl Med 2001; 42:1062-76.

48. Merlet P, Valette H, Dubois-Rande JL, Moyse D, Duboc D, Dove $\mathrm{P}$, et al. Prognostic value of cardiac metaiodobenzylguanidine imaging in patients with heart failure. J Nucl Med 1992;33:471-7.

49. Verberne HJ, Brewster LM, Somsen GA, van Eck-Smit BL. Prognostic value of myocardial ${ }^{123}$ Imetaiodobenzylguanidine (MIBG) parameters in patients with heart failure: A systematic review. Eur Heart J 2008;29:1147-59.

50. Higuchi T, Schwaiger M. Imaging cardiac neuronal function and dysfunction. Curr Cardiol Rep 2006;8:131-8.

51. Wakasugi S, Fischman AJ, Babich JW, et al. Metaiodobenzylguanidine: Evaluation of its potential as a tracer for monitoring doxorubicin cardiomyopathy. J Nucl Med 1993;34:1282-6.

52. Valdes Olmos RA, ten Bokkel Huinink ww, ten Hoeve RF, van Tinteren H, Bruning PF, can Vlies B, et al. Assessment of anthracycline-related myocardial adrenergic derangement by [123I]metaiodobenzylguanidine scintigraphy. Eur J Cancer 1995;31A:26-31.

53. Jacobson AF, Senior R, Cerqueira MD, Wong ND, Thomas GS, Lopez VA, et al. Myocardial iodine-123 meta-iodobenzylguanidine imaging and cardiac events in heart failure results of the prospective ADMIRE-HF (AdreView Myocardial Imaging for Risk Evaluation in Heart Failure) Study. J Am Coll Cardiol 2010;55:2212-21.

54. Carrio I, Estorch M, Berna' L, et al. ${ }^{111}$ In-antimyosin and ${ }^{123} \mathrm{I}-$ MIBG studies in the early assessment of doxorubicin cardiotoxicity. J Nucl Med 1995;36:2044-9.

55. Bengel FM, Schwaiger M. Assessment of cardiac sympathetic neuronal function using PET imaging. J Nucl Cardiol 2004;11: 603-16.

56. Carrio I, Lopez-Pousa A, Estorch M, et al. Detection of doxorubicin cardiotoxicity in patients with sarcomas by indium-111antimyosin monoclonal antibody studies. J Nucl Med 1993;34: 1503-7.

57. Kremer LCM, Tiel-van Buul MMC, Ubbink MC, Offringa M, Ottenkamp J, Olmos RV, et al. Indium-111-antimyosin scintigraphy in the early detection of heart damage after anthracycline therapy in children. J Clin Oncol 1999;17:1208-11.

58. Bennink RJ, van den Hoff MJ, can Hemert FJ, de Bruin KM, Spijkereboer AL, van den Vanderheyden J, et al. Annexin V imaging of acute doxorubicin cardiotoxicity (apoptosis) in rats. J Nucl Med 2004;45:842-8.

59. Kalyanaraman B, Joseph J, Kalivendi S, Wang S, Konorev E, Kotamraju S. Doxorubicin induced apoptosis: Implications in cardiotoxicity. Mol Cell Biochem 2002;234-235:119-24.

60. Takemura G, Fujiwara H. Doxorubicin-induced cardiomyopathy: From the cardiotoxic mechanisms to management. Prog Cardiovasc Dis 2007;49:330-52.

61. Saito K, Takeda K, Imanaka-Yoshida K, Imai H, Sekine T, Kamikura Y. Assessment of fatty acid metabolism in taxaninduced myocardial damage with iodine-123 BMIPP SPECT: Comparative study with myocardial perfusion, left ventricular function, and histopathological findings. Ann Nucl Med 2003; 17:481-8.
62. de Korte MA, de Vries EG, Lub-de Hooge MN, et al. ${ }^{111}$ Indiumtrastuzumab visualises myocardial human epidermal growth factor receptor 2 expression shortly after anthracycline treatment but not during heart failure: A clue to uncover the mechanisms of trastuzumab-related cardiotoxicity. Eur J Cancer 2007;43:2046-51.

63. Cardinale D, Sandri MT. Role of biomarkers in chemotherapyinduced cardiotoxicity. Prog Cardiovasc Dis 2010;53:121-9.

64. Kalay N, Basar E, Ozdogru I, Er O, Cetinkaya Y, Dogan A, et al. Protective effects of carvedilol against anthracycline-induced cardiomyopathy. J Am Coll Cardiol 2006;48:2258-62.

65. Roziakova L, Bojtarova E, Mistrik M, Dubrava J, Gergel J, Lenkova N, et al. Serial measurements of cardiac biomarkers in patients after allogeneic hematopoietic stem cell transplantation. J Exp Clin Cancer Res 2012;9:13. doi:10.1186/1756-9966-31-13.

66. Fallah-Rad N, Walker JR, Wassef A, Lytwyn M, Bohonis S, Fang T, et al. The utility of cardiac biomarkers, tissue velocity and strain imaging, and cardiac magnetic resonance imaging in predicting early left ventricular dysfunction in patients with human epidermal growth factor receptor II-positive breast cancer treated with adjuvant traztuzumab therapy. J Am Coll Cardiol 2011:57:2263-70.

67. Dodos F, Halbsguth T, Erdmann E, Hoppe UC. Usefulness of myocardial performance index and biochemical markers for early detection of anthracycline-induced cardiotoxicity in adults. Clin Res Cardiol 2007;97:318-26.

68. Dolci A, Dominici R, Cardinale D, Sandri MT, Panteghini M. Biochemical markers for prediction of chemotherapy-induced cardiotoxicity systematic review of the literature and recommendations for use. Am J Clin Pathol 2008;130:688-95.

69. Visscher H, Ross CJ, Rassekh SR, Barhdadi A, Dubé MP, Al-Saloos $\mathrm{H}$, et al. Pharmacogenomic prediction of anthracyclineinduced cardiotoxicity in children. J Clin Oncol 2012;30:1422-8.

70. Lenihan DJ, Fisch M. Effectiveness of using biomarkers to detect and identify cardiotoxicity and describe treatment (PREDICT). http://clinicaltrials.gov/ct2/show/NCT01311843. Accessed 30 Mar 2013.

71. Jurcut R, Wildiers H, Ganame J, D'hooge J, De Backer J, Denys $\mathrm{H}$, et al. Strain rate imaging detects early cardiac effects of pegylated liposomal Doxorubicin as adjuvant therapy in elderly patients with breast cancer. J Am Soc Echocardiogr 2008;21: 1283-9.

72. Ho E, Brown A, Barrett P, Morgan RB, King G, Kennedy MJ, et al. Subclinical anthracycline- and trastuzumab-induced cardiotoxicity in the long-term follow-up of asymptomatic breast cancer survivors: A speckle tracking echocardiographic study. Heart 2010;96:701-7.

73. Sawaya H, Sebaq IA, Plana JC, Januzi JL, Ky B, Gosavi S, et al. Early detection and prediction of cardiotoxicity in chemotherapytreated patients. Am J Cardiol 2011;107:1375-80.

74. Sawaya H, Sebag IA, Plana JC, Januzzi JL, Ky B, Tan TC, et al. Assessment of echocardiography and biomarkers for the extended prediction of cardiotoxicity in patients treated with anthracyclines, taxanes, and trastuzumab. Circ Cardiovasc Imaging 2012;5:596-603.

75. Jiji RS, Kramer CM, Salerno M. Non-invasive imaging and monitoring cardiotoxicity of cancer therapeutic drugs. J Nucl Cardiol 2012;19:377-88.

76. Lipschultz SE, Cochran TR, Wilkinson JD. Screening for longterm cardiac status during cancer treatment. Circ Cardiovasc Imaging 2012;5:555-8.

77. Tantawy AA, Elmasry OA, Shaaban M, Toaima DN, El Shahat AM. Radionuclide ventriculography detects early anthracycline cardiotoxicity in children with Hodgkin lymphoma. J Pediatr Hematol Oncol 2011;33:e132-7. 
78. Poutanen T, Tikanoja T, Riikonen P, Silvast A, Perkkio M. Longterm prospective follow-up study of cardiac function after cardiotoxic therapy for malignancy in children. J Clin Oncol 2003;21:2349-56.

79. Pratt CB, Ransom JL, Evans WE. Age-related Adriamycin cardiotoxicity in children. Cancer Treat Rep 1978;62:1381-5.

80. Mulrooney DA, Yeazel MW, Kawashina T, Mertens AC, Mitby $\mathrm{P}$, Stovall $\mathrm{M}$, et al. Cardiac outcomes in a cohort of adult survivors of childhood and adolescent cancer: Retrospective analysis of the Childhood Cancer Survivor Study cohort. BMJ 2009;339:b4606.

81. Beeres SLMA, Bengel FM, Bartunek J, Atsma DE, Hill JM, Vanderheyden $\mathrm{M}$, et al. Role of imaging in cardiac stem cell therapy. J Am Coll Cardiol 2007;49:1137-48.

82. ACCF/ACR/SCCT/SCMR/ASNC/NASCI/SCAI/SIR. Appropriateness criteria for cardiac computed tomography and cardiac magnetic resonance imaging. J Am Coll Cardiol 2006;48:1475-97.

83. Zagrosek A, Abdel-Aty H, Boye P, Wassmuth R, Messroghli D, Utz W, et al. Cardiac magnetic resonance monitors reversible and irreversible myocardial injury in myocarditis. J Am Coll Cardiol Imaging 2009;2:131-8.

84. Fallah-Rad N, Lytwyn M, Fang T, Kirkpatrick I, Jassal DS. Delayed contrast enhancement cardiac magnetic resonance imaging in traztuzumab induced cardiomyopathy. J Cardiovasc Mag Res 2008;10:5.

85. Wassmuth R, Lentszch S, Erdbruegger U, Schulz-Menger J, Doerken B, Dietz R, et al. Subclinical cardiotoxic effects of anthracyclines as assessed by magnetic resonance imaging-a pilot study. Am Heart J 2001;141:1007-13.

86. Lightfoot JC, D'Agostino RB Jr, Hamilton CA, Jordan J, Torti FM, Kock ND, et al. Novel approach to early detection of doxorubicin cardiotoxicity by gadolinium-enhanced cardiovascular magnetic resonance imaging in an experimental model. Circ Cardiovasc Imaging 2010;3:550-8.

87. Westwood M, Anderson LJ, Firmin DN, et al. A single breathhold multiecho $\mathrm{T} 2 *$ cardiovascular magnetic resonance technique for diagnosis of myocardial iron overload. J Magn Reson Imaging 2003;18:33-9.

88. Dash R, Chung J, Chan T, Yamada M, Barral J, Nishimura D, et al. A molecular MRI probe to detect treatment of cardiac apoptosis in vivo. Magn Reson Med 2011;66:1152-62.

89. Steel K, Broderick R, Gandla V, Larose E, Resnic F, JeroschHerold $\mathrm{M}$, et al. Complementary prognostic values of stress myocardial perfusion and late gadolinium enhancement imaging by cardiac magnetic resonance in patients with known or suspected coronary artery disease. Circulation 2009;120:1390-400.
90. Azevedo CF, Nigri M, Higuchi ML, Pomerantzeff PM, Spina GS, Sampaio RO, et al. Prognostic significance of myocardial fibrosis quantification by histopathology and magnetic resonance imaging in patients with severe aortic valve disease. J Am Coll Cardiol 2010;56:278-87.

91. Bruder O, Wagner A, Jensen CJ, Schneider S, Ong P, Kispert EM, et al. Myocardial scar visualized by cardiovascular magnetic resonance imaging predicts major adverse events in patients with hypertrophic cardiomyopathy. J Am Coll Cardiol 2010;56:87587.

92. Adabag AS, Maron BJ, Appelbaum E, Harrigan CJ, Buros JL, Gibson CM, et al. Occurrence and frequency of arrhythmias in hypertrophic cardiomyopathy in relation to delayed enhancement on cardiovascular magnetic resonance. J Am Coll Cardiol 2008;51:1369-74.

93. Patel MR, Cawley PJ, Heitner JF, Klem I, Parker MA, Jaroudi WA, et al. Detection of myocardial damage in patients with sarcoidosis. Circulation 2009;120:1969-77.

94. Mekinian A, Lions C, Leleu X, Duhamel A, Lamblin N, Coiteux $\mathrm{V}$, et al. Prognosis assessment of cardiac involvement of systemic AL amyloidosis by magnetic resonance imaging. Am J Med 2010;123:864-8

95. Thorburn A, Frankel AE. Apoptosis and anthracycline cardiotoxicity. Mol Cancer Ther 2006;5:197-9.

96. Horie T, Ono K, Nishi H, Nagao K, Minako K, Watanable S, et al. Acute doxorubicin cardiotoxicity is associated with miR146a-induced inhibition of the neuregulin-ErbB pathway. Cardiovasc Res 2010;87:656-64.

97. Shi J, Zhang L, Zhang Y, Surma M, Payner RM, Wei L. Downregulation of doxorubicin-induced myocardial apoptosis accompanies postnatal heart maturation. Am J Physiol Heart Circ Physiol 2012. doi:10.1152/ajpheart.00844.2011.

98. Arai M, Yoguchi A, Takizawa T, Yokoyama T, Kanda T, Kurabayashi M, et al. Mechanism of Doxorubicin-induced inhibition of sarcoplasmic reticulum $\mathrm{Ca}^{2+}$-ATPase gene transcription. Circ Res 2000;86:8-14.

99. Sadeghi M, Schwartz RG, Beanlands RS, Al-Mallah MH, Bengel FM, Borges-Neto S, et al. for the Cardiovascular Council Board of Directors of the Society of Nuclear Medicine. Cardiovascular nuclear imaging: Balancing proven clinical value and potential radiation risk. J Nucl Med 2011;52:1163-4.

100. Lal H, Kolaja KL, Force T. Cancer genetics and the cardiotoxicity of the therapeutics. J Am Coll Cardiol 2013;61:267-74.

101. Panjrath GS, Jain D. Imaging cancer chemotherapy-induced cardiotoxicity. J Nucl Cardiol 2006;13:415-26. 